\title{
Wirtschaft und Gesellschaft: neomarxistische Theorieansätze
}

Stefan Kühl

\section{Einleitung}

Mitte der achtziger Jahre des vergangenen Jahrhunderts konnte Richard Swedberg (1987) noch behaupten, dass der Neo-Marxismus wie eine ,frische Brise' die eher ,muffige' Wirtschaftssoziologie erfasst hätte. Swedbergs Einschätzung war nicht überraschend. Besonders in den siebziger Jahren erschienen eine Vielzahl durch den Marxismus inspirierte Werke. Marxistische Ansätze wie die Regulationstheorie, die Dependenztheorie, die Weltsystemtheorie oder die Labour Process Debate beeinflussten nicht nur die Wirtschaftssoziologie, sondern häufig auch die allgemeine Soziologie (vgl. Heinemann 1987: 13). ${ }^{1}$ Zwanzig Jahre nach der fast euphorischen Einschätzung Swedbergs scheint in weiten Teilen der Wirtschaftssoziologie Marx kaum noch eine Rolle zu spielen. Bei einer Sichtung wirtschaftssoziologischer Übersichtsbeiträge entsteht der Eindruck, als wenn Marxismus nur noch als historische Traditionslinie oder als Abgrenzungsfolie relevant ist. Marx wird als Klassiker auf den ersten Seiten kurz erwähnt, spielt dann aber in der Darstellung der aktuellen Forschungsrichtungen keine Rolle mehr. Grundbegriffe von Marx wie Ware, Arbeitskraft, absoluter und relativer Mehrwert, formale und reelle Subsumtion sind weitgehend aus den wirtschaftssoziologischen Darstellungen verschwunden. Die aktuellen marxistischen Forschungsrichtungen spielen eine geringe Rolle und werden durch system-, governance-, strukturationstheoretische oder institutionenökonomische Elemente ersetzt (vgl. Weber, Wegge 2003; Jansen 2005; Hirsch-Kreinsen 2005). ${ }^{2}$

Vieles erinnert heute an die sechziger Jahre, als gerade unter den amerikanischen Wirtschaftssoziologen tabuisiert zu sein schien, die ältere Generation der Marxisten wie Antonio Gramsci, Rudolf Hilferding, Georg Lukacs oder Rosa Luxemburg überhaupt noch zu zitieren. Nicht zuletzt auch durch den einsetzenden Kalten Krieg beeinflusst, schaffte es Karl Marx in den sechziger Jahren häufig noch nicht einmal auf die Leseliste linker Parsonianer wie Wilbert E. Moore und Neil Smelser (vgl. Swedberg 1987: 79). Das Ziel dieses Beitrages

\footnotetext{
${ }^{1}$ In einer Umfrage unter amerikanischen Wirtschaftssoziologen Anfang der achtziger Jahre gab die überwältigende Mehrzahl von diesen an, mit marxistischen oder neomarxistischen Ansätzen zu arbeiten (vgl. Makler et al. 1982). Charakteristisch für die deutschsprachige Renaissance von Marx in der Wirtschaftssoziologie ist die Darstellung in den Lehrbüchern (vgl. z. B. Türk 1987).

${ }^{2}$ Interessant ist auch die (Nicht-)Behandlung marxistischer Theorien in der überarbeiten Auflage des Handbook of Economic Sociology (Smelser, Swedberg 2005). In den einzelnen Beiträgen erscheint Marx, wenn überhaupt, in den ersten zwei Seiten als ,classical legacy' (vgl. z. B. King, Szelényi 2005: 207; Stearns, Mizruchi 2005: 284; Abbott 2005: 307; Wuthnow 2005: 604). Neomarxistische Theorien werden, wenn überhaupt, mit einzelnen Querverweisen abgehandelt (z. B. Dependenztheorie, Weltsystemtheorie und Regulationstheorie im Artikel von Gereffi 2005: 166 f. und die Weltsystemtheorie im Artikel von Fligstein 2005: 189).
} 
ist es, gegen diesen Trend zur ,Entmarxisierung der Wirtschaftssoziologie' zentrale Argumente einer durch den Marxismus geprägten neueren Forschungstradition darzustellen und einzuordnen. ${ }^{3}$ In den Mittelpunkt der Darstellung stelle ich dabei die Versuche der neomarxistischen Ansätze, eine marxistisch inspirierte Wirtschaftstheorie zu einer Gesellschaftstheorie auszubauen. Letztlich stehen alle neomarxistischen Ansätze insofern in der Tradition von Marx, als es nicht nur um die Beschreibung der kapitalistischen Wirtschaft, sondern um die Beschreibung der kapitalistischen Gesellschaft geht. „In allen Gesellschaftsformen“, so bereits Marx, „ist es eine bestimmte Produktion“, die allen übrigen Verhältnissen „Rang und Einfluß anweist“. „Das Kapital ist die alles beherrschende Macht der bürgerlichen Gesellschaft.“ Es muss deswegen "Ausgangspunkt wie Endpunkt" der Analyse bilden (vgl. Marx 1961: 637). Im Kapitalismus werde, so Marx und Engels, die Gesellschaft in einer noch durchdringenderen Weise als im Feudalismus von der Wirtschaft ,vereinnahmt'. „Die Bourgeoisie“ könne nicht existieren, ohne „die Produktionsverhältnisse, also sämtliche gesellschaftliche Verhältnisse, fortwährend zu revolutionieren“ (Marx, Engels 1959 [1948]: 465).

Die simplifizierende Formel, mit dem der Nexus zwischen Wirtschaft und Gesellschaft von Marx hergestellt wurde, war das Primat der Ökonomie: Die ökonomische Basis, so die bekannte Kurzformel, bestimmt den Überbau (vgl. Marx 1961 [1859]: 8). ${ }^{4}$ Ganz im Sinne dieser Formel konzipierten Marx und Engels in ihren Frühschriften Politik und Recht noch vordringlich als ein Instrument des Kapitals, um die ökonomische Ausbeutung der Arbeiter fortsetzen zu können. Recht war aus dieser Perspektive für Marx und Engels nur „der zum Gesetz erhobene Wille einer Klasse“. Politik stellte „die organisierte Gewalt einer Klasse zur Unterdrückung einer anderen“ dar (Marx, Engels 1959 [1948]: 477). Dieses Bild wurde aber schon von Marx und Engels immer stärker modifiziert, durch Marx besonders in den Grundrissen der Kritik der politischen Ökonomie und in seiner Analyse des französischen Staates unter Louis Napoleon (vgl. Marx 1960 [1851]; 1961 [1859]), durch Engels besonders in seinen Überlegungen zum Ursprung der Familie, des Privateigentums und des Staates (vgl. Engels 1969). Ohne dass Karl Marx und Friedrich Engels jemals eine theoretisch kohärente Staatstheorie herausgearbeitet hätten, wird bei beiden deutlich, dass sie sowohl Politik als auch Recht als eher autonome Autoritäten begriffen, die als ,Regulierer' des Klassenkampfes eine stabilisierende Funktion für den Kapitalismus übernehmen. Dabei befinden sich Recht und Politik in einer Zwitterposition. Auf der einen Seite repräsentieren sie die Herrschaftsverhältnisse von Kapital und Arbeit, auf der anderen Seite nehmen sie aber auch die Funktion eines klassenübergreifenden Regulators des Klassenkampfes wahr. Während die Ausarbeitung einer anspruchsvollen Wirtschaftstheorie von Karl Marx in vielen Aspekten als gelungen betrachtet werden kann, blieb der Nexus zwischen Wirtschafts- und Gesellschaftstheorie weitgehend unausgearbeitet. ${ }^{5}$ Sichtbar wird dies beispielsweise an der weitgehend fehlenden Klassen- und Staatstheorie von Marx.

\footnotetext{
${ }^{3}$ Der Beitrag konzentriert sich auf die neomarxistischen Theoriestränge, die für die Wirtschaftssoziologie besonders relevant sind. Das erklärt die Nichtbehandlung beispielsweise der kritischen Theorie und der marxistischen Praxisphilosophie.

${ }^{4}$ Auf diesen Nexus wird auch immer wieder in wirtschaftssoziologischen Grundlagenwerken verwiesen (vgl. Fürstenberg 1970: 122 f.; Buß 1985: 30 ff.; Hillmann 1988: 13 f.).

${ }^{5}$ Der umstrittenste und für die Wirtschaftssoziologie relevante Streitpunkt ist sicherlich nach wie vor die Marx'sche Arbeitswertlehre (vgl. Hofmann 1971; Helmedag 1992).
} 
Dieser Artikel konzentriert sich auf vier neomarxistische Theorierichtungen, die für die wirtschaftssoziologische Debatte von besonderer Bedeutung sind: die Regulationstheorie (Abschnitt 2), die Dependenz- und Weltsystemtheorie (Abschnitt 3), die durch Marx beeinflusste(n) Klassentheorie(n) (Abschnitt 4) sowie die Labour Process Debate (Abschnitt 5). ${ }^{6}$ In allen diesen Theorien ist die Bemühung zu beobachten, eine auf die Wirtschaft konzentrierte Politökonomie zu einer Gesellschaftstheorie auszubauen. Dieser Sprung von einer Wirtschafts- zu einer Gesellschaftstheorie ist, so meine These, jedoch nur begrenzt gelungen. Alternativ lässt sich, so mein Vorschlag, die Marx'sche Wirtschaftstheorie als eine Theorie der Ausdifferenzierung des Wirtschaftssystems lesen - mit parallel dazu auftretenden Ausdifferenzierungsprozessen anderer gesellschaftlicher Teilbereiche (Abschnitt 6). ${ }^{7}$

\section{Regulationstheorie}

Zentrale Herausforderung für eine neomarxistisch orientierte Wirtschaftssoziologie war es, die Entwicklung des Kapitalismus als ein zentrales strukturierendes Merkmal moderner Gesellschaften in den Griff zu bekommen. Wie kann man aus einer marxistischen Perspektive erklären, dass es dem Kapitalismus trotz der immer wieder auftretenden Krisen gelingt, zu überleben? Durch welches ,Flickwerk' und welche ,partikularen Maßnahmen' gelingt es, den Gegensatz von Kapital und Arbeit stabil zu halten (vgl. Adorno 1969: 20)? Welche gesellschaftlichen Strukturen sind es, die die fortlaufende kapitalistische Produktion sicherstellen, und wie entstehen und transformieren sie sich (vgl. Aglietta 1979: 17)?

Der Regulationsansatz, der aus der politischen Ökonomie kommt, aber wie kaum eine andere Theorie die an Gesellschaftsfragen interessierte Wirtschaftssoziologie beeinflusst hat, geht davon aus, dass die Dynamik des Kapitals eine ,gigantische Produktivkraft freisetzt', das Kapital aber gleichzeitig eine ,blinde Macht' ist, die die freigesetzten Kräfte selbst nicht bändigen kann. Die kapitalistische Wirtschaft hat, so die Annahme der Regulationstheoretiker, die Fähigkeit, menschliche Energien so in Bewegung zu setzen, dass sie in Wachstum verwandelt werden. Sie sei jedoch nicht in der Lage, angesichts der aufeinander prallenden Einzelinteressen selbst den Gesamtzusammenhalt zu gewährleisten (vgl. Aglietta 2000: 19). ${ }^{8}$ Erst der Blick auf die Ausbildung von ökonomischen, politischen, rechtlichen und sozialen Regulationsweisen könne erklären, weswegen der Kapitalismus trotz immer wieder auftretender Krisen am Leben bleibt. Die Aushandlungen zwischen den Vertretungsorganen von Kapital und Arbeit, die staatlichen Instanzen der Politik, ein sowohl von der Politik als auch von der Wirtschaft weitgehend autonomes Rechtssystem, die verschiedenen schulischen

\footnotetext{
${ }^{6}$ Ich verzichte aus Platzgründen auf die Darstellung von solchen wirtschaftssoziologischen Ansätzen, die als Ein- oder Zweipersonen-Unternehmung entstanden sind und (noch) keine größere Schulenbildung nach sich gezogen haben (vgl. z. B. Offe 1972; Boltanski, Chiapello 1999; Kurz 2001; Hardt, Negri 2003). Teilweise wird bei der Darstellung der neomarxistischen Schulen auf diese Ansätze verwiesen. Auf die wirtschaftssoziologisch relevante Theorie des Monopolkapitalismus (vgl. Baran, Sweezy 1966) wird verzichtet, weil meines Erachtens nur noch sehr vereinzelt auf diese Theorie Bezug genommen wird.

${ }^{7}$ Die Darstellung der Regulationstheorie, der Klassentheorie und der Labour Process Debate basiert auf der Präsentation in meinem Lehrbuch (vgl. Kühl 2004), das versucht die Erklärungskraft von Marxismus und Systemtheorie für zentrale Themen der Arbeits-, Wirtschafts-, Industrie- und Betriebssoziologie zu prüfen.

${ }^{8}$ Eine deutsche Spielart der Regulationstheorie stellt Claus Offes (1972; 1984; 2006) Analyse des Spätkapitalismus dar.
} 
und universitären Bildungseinrichtungen, die wissenschaftlichen Institutionen und die Massenmedien trügen, so die Auffassung der Regulationstheoretiker, dazu bei, den ,Klassenkampf' zu kanalisieren, ohne die weitere Akkumulation von Kapital zu gefährden (vgl. Aglietta 1979: 123; Lipietz, Krebs 1998: 17; Aglietta 2000: 19; Jessop 2003: 91).

Wenn sich eine dominierende Regulationsweise ausgebildet hat, könne es, so die Auffassung der Regulationstheoretiker, immer wieder relativ stabile Phasen des Kapitalismus geben. Es träten lediglich kleinere Krisen auf, die die grundlegende Regulationsweise nicht bedrohen. Aber aufgrund des Strebens des Kapitals nach immer höheren Profiten könnten sich die Investitionsstrategien, die Produktionstechnologien und die Arbeitsorganisationen so verändern, dass sie in Widerspruch zu den herrschenden Formen der Regulation gerieten. Mit der Zeit entstünden strukturelle Krisen, die dazu führten, dass die alte Regulationsweise zusammenbreche und sich eine neue kapitalistische Regulationsweise durchsetze. Auf dieser Basis entwickeln die Regulationstheoretiker ein Vierphasenmodell, mit dem die Entwicklung des Kapitalismus bis heute erklärt werden soll. In der ersten Phase bis zur Mitte des neunzehnten Jahrhunderts war in den Vereinigten Staaten und in weiten Teilen Europas der Übergang von der Feudalgesellschaft zum Kapitalismus weitgehend abgeschlossen. In dieser Frühphase des entwickelten Kapitalismus investierten die Unternehmer ihr Kapital vorwiegend in die Verbesserung der bestehenden Produktionsanlagen. Natürlich wurden neue technische Entwicklungen besonders im Bereich der Werkzeugmaschinen, des Dampfantriebes und der Gusstechniken aufgegriffen und in die Produktion integriert, aber die Unternehmen versuchten vor allem, das bereits vorhandene Wissen für ihre Geschäfte zu nutzen. Die Profitmaximierungsstrategie der Unternehmen war, so die Analyse der Regulationstheoretiker, darauf gerichtet, durch die Verlängerung des Arbeitstages noch mehr aus der Arbeitskraft der Lohnarbeiter herauszuholen. Michel Aglietta bezeichnet diese auf Lohnsenkung und Arbeitszeitverlängerung ausgerichtete Haltung des Kapitals als eine „extensive Akkumulationsstrategie“ (vgl. Aglietta 1979: 130; Glick, Brenner 1999: 43). Warum investierten in dieser Frühphase des entwickelten Kapitalismus die Unternehmen nur begrenzt in die Produktionstechniken? Eine zentrale Ursache lag nach Auffassung der Regulationstheoretiker in der mangelnden Nachfrage auf den Konsummärkten. Bis in die Mitte des neunzehnten Jahrhunderts sicherten sich viele Arbeiter ihr Überleben durch Selbstversorgung und nicht durch die Waren, die sie sich mit ihrem Lohn kauften. Die Gruppe der Kleinbauern, Heimarbeiter und ländlichen Handwerker, teilweise aber auch die städtischen Handwerker und Arbeiter besaßen oder pachteten ein kleines Stück Land und bauten dort Nahrungsmittel für den Eigenbedarf an. Diese Subsistenzwirtschaft war für die Arbeiter häufig wichtiger als die nur unregelmäßige und auf wenige Monate im Jahr beschränkte Lohnarbeit (vgl. Kocka 1983: 40 ff.). Dies machte die Arbeiter ein Stück weit unabhängig von Schwankungen auf den Warenmärkten, ermöglichte es dem Kapital andererseits aber auch, sehr niedrige Löhne zu bezahlen. Die Regulationstheoretiker bezeichnen diesen Prozess, der die Frühphase des Kapitalismus bestimmte, als „Dominanz nichtwarenförmiger Verhältnisse“ (gemeint ist die Subsistenzwirtschaft) über „warenförmige Verhältnisse in der Konsumtionsweise“ (Aglietta 1979: 80).

In der zweiten Phase zu Beginn des zwanzigsten Jahrhunderts setzte sich schrittweise die Massenproduktion als zentrale Strategie des Kapitals durch. Die Massenproduktion, zentraler Aspekt der durch Elektrifizierung, Verbreitung des Verbrennungsmotors und 
Ausbreitung der chemischen Industrie geprägten zweiten industriellen Revolution (Friedmann 1959: 6 ff.), erforderte enorme Investitionen des Kapitalisten in Förderanlagen, Maschinen und Produktionstechniken. Die auf Technologieentwicklung und Investition in Maschinen gestützte Strategie wird von den Regulationstheoretikern als ,intensive Akkumulationsstrategie' des Kapitals identifiziert. Die enormen Investitionen in die Produktionsanlagen konnten sich viele Unternehmen nicht leisten, und in wichtigen Branchen kam es deswegen zu einer Ausbildung von Oligopolen, einer Ansammlung von wenigen Unternehmen, die den Markt weitgehend unter sich aufteilen konnten.

Das auf Massenproduktion basierende kapitalistische System wird unter Rückgriff auf eine Begriffsschöpfung des sozialistischen Theoretikers Antonio Gramsci als Fordismus bezeichnet (vgl. Aglietta 1979: 117; Gramsci 1999: 2061 ff.; Tanner 1999: 480 ff.). Henry Ford war der Gründer der Ford-Automobilwerke und eine der treibenden Kräfte bei der Einführung des Prinzips der Massenproduktion. Werkzeuge und Arbeiter sollten in der „Reihenfolge der bevorstehenden Verrichtungen" angeordnet werden, so dass jedes Teil während des Produktionsprozesses einen möglichst kurzen Weg zurücklegte (vgl. Ford 1923: 93). Die große kapitalistische Herausforderung bestand in dieser zweiten Phase darin, dass die Massenproduktion von Konsumgütern nicht auf eine entsprechende Nachfrage stieß. Die große Wirtschaftskrise am Ende der zwanziger Jahre des zwanzigsten Jahrhunderts wird von den Regulationstheoretikern als Indiz dafür gesehen, dass die sich ausbildenden neuen fordistischen Produktionsformen zwar enorme Effizienzvorteile brachten, dies jedoch zu einer Krise führte, da für die Produkte keine zahlungskräftigen Abnehmerschichten vorhanden waren. Das Problem war, um die Terminologie der Regulationstheoretiker zu nutzen, das eines „intensiven Akkumulationsregimes ohne Massenkonsum“ (Lipietz 1985: 123 f.; Lipietz, Krebs 1998: 38 f.). In fast paradoxer Weise trugen, so die Auffassung der Regulationstheoretiker, erst die erfolgreichen Kämpfe der Arbeiterklassen für eine Verbesserung ihrer Lebensbedingungen dazu bei, dass sich ansatzweise bereits nach der Weltwirtschaftskrise der späten zwanziger Jahre, aber besonders nach dem Zweiten Weltkrieg ein stabiler Markt für Massenkonsumprodukte ausbildete. Erst als die Kapitalisten gezwungen waren, den Arbeitern höhere Löhne zu bezahlen, bildeten sich Märkte, die eine neue, stabile Entwicklungsstufe des Kapitalismus ermöglichten (vgl. Aglietta 1979: 154 ff.).

Der große fordistische Kompromiss, der sich in der dritten Phase nach dem Zweiten Weltkrieg abzeichnete, sah so aus, dass die Profite, die aus den Effizienzgewinnen der fordistischen Produktionsform gezogen wurden, nicht nur für Investitionen in neue Maschinen genutzt wurden, sondern auch in Form steigender Löhne an die Arbeiter weitergegeben wurden. Diese Lohnsteigerungen bildeten nach Ansicht der Regulationstheoretiker die Basis für den Massenkonsum in den westlichen Industriestaaten, an dessen Ende sich fast jeder Arbeitnehmer seinen Kleinwagen, seine Waschmaschine und seinen Mallorcaurlaub leisten konnte (vgl. Aglietta 2002: 13). Fordismus bezeichnet für die Regulationstheoretiker also letztlich nicht nur eine auf Gleitbahnen, Fließbändern und anderen Transportmitteln basierende Form der Produktion, sondern ebenso eine grundlegende Veränderung der Konsummuster der arbeitenden Bevölkerung. Wie keine andere Aussage steht eine Äußerung Henry Fords für diese doppelte Orientierung auf Produktion und Konsum: Jeder seiner Arbeiter, so Ford, sollte sich auch das in seinen Werken produzierte Auto, die Tin Lizzy, leisten können. 
Welche Rolle spielte der Staat bei der Ausbildung dieser stabilen Produktions- und Konsumtionsverhältnisse? In der Tradition von Marx gehen die Regulationstheoretiker davon aus, dass das Kapital von sich aus kein Interesse daran hat, die Überproduktions- oder besser die Unterkonsumptionskrise durch ein Anheben des Lohnniveaus der Arbeiter zu lösen. Erst ein ,etatistischer Reformismus' habe, so die Argumentation der Regulationstheoretiker, die Anhebung des Lohnniveaus ermöglicht. Diese hochgradig standardisierte Form von Arbeit und Massenkonsum, die ,innere Landnahme' immer mehr gesellschaftlicher Bereiche durch die industriellen Produktionsparadigma und die Integrationswirkung des Sozialstaates kumulierten bei einigen Beobachtern in der Diagnose einer ,formierten Gesellschaft' (Ludwig Ehrhard) oder ,programmierten Gesellschaft” (Alain Touraine). Das „Leben der Menschen“ im fordistischen Zeitalter wurde, so Ulrich Beck, „ebenso standardisiert wie die Stahlbleche, aus denen sie Autos zusammenschweißten“ (Beck 2000: 39). Aber der „Traum der immerwährenden Prosperität“ (Lutz 1989), die Hoffnung auf ein andauerndes "goldenes Zeitalter" (Lipietz, Krebs 1998) währte nur kurz. Der Fordismus führte, so die Diagnose der Regulationstheoretiker, über kurz oder lang zu sinkenden Profitraten. Die Vorteile, die das Management aus der Intensivierung der Arbeit, aus der Zerstückelung des Arbeitsprozesses und der Einführung neuer Maschinen gezogen hatte, hatten sich erschöpft. Zusätzlich war die vorrangig nationalstaatliche Regulationsweise in Widerspruch zur zunehmenden Internationalisierung der Produktion geraten (vgl. Aglietta 1979: 163; Lipietz, Krebs 1998: 39; Hirsch 1995: 84 ff.).

Aus diesen Krisenerscheinungen ergab sich lange Zeit das Forschungsprogramm für die Wirtschaftssoziologie: Welche Konturen hat die kapitalistische Gesellschaft nach dem Ende des dominierenden fordistischen Zeitalters? Bei der Beschreibung der sich nach dem Fordismus ausbildenden kapitalistischen Gesellschaftsordnung in der vierten Phase tendieren die in der Marx'schen Tradition stehenden Soziologen entweder zu der sehr abstrakten Formulierung des ,Postfordismus', ,Spätfordismus' oder ,Spätkapitalismus' (vgl. z. B. Hirsch, Roth 1986; früh schon Offe 1972) oder sie versuchen über Konzepte wie „Toyotisierung“ (Roth 1994), "Sonyismus“ (Wark 1991) oder dem sich aus Windows und Intel zusammensetzenden „Wintelismus“ (Borrus, Zysman 1998) einen möglichst präzisen Gegenbegriff zum Fordismus zu formulieren. Während in der ersten Begriffsstrategie schon allein das ,post' und das ,spät' eine gewisse Hilflosigkeit bei der Bestimmung der neuen Regulationsweise zum Ausdruck bringen, hat die Begriffswahl des ,Toyotismus', ,Sonyismus' oder ,Wintelismus' häufig zu einer Einengung der Perspektive auf die reinen Produktionsstrategien des Kapitals geführt. ${ }^{9}$ Weitgehend Einigkeit bei Regulationstheoretikern besteht darin, dass man sich nicht einig ist, worin die neue Regulationsweise des ,Postfordismus', des ,Toyotismus', des ,Sonyismus' oder des ,Wintelismus' genau besteht. Die genaue Bestimmung für die Regulationsweise des Fordismus konnte für die heutige Zeit bisher von den Regulationstheoretikern nicht wiederholt werden. Es zeichnen sich lediglich zwei zentrale Trends auf, aus denen sich auf die Konturen des Postfordismus schließen lässt: die zunehmende Bedeutung globaler Finanzmärkte und die Entstehung globaler Wertschöpfungsketten.

\footnotetext{
${ }_{9}$ Anders als im Begriff des Fordismus, der in der ursprünglichen Anlage einen gesellschaftstheoretischen Ansatz formulierte, gerieten die unter den Begriffen des ,Toyotismus', ,Sonyismus' oder ,Wintelismus' zusammengefassten Studien häufig zu fast theorieabstinenten betriebssoziologischen Studien (vgl. die Kritik von Hübner, Mahnkopf 1988: 24 f.; Jessop 2003: 95 ff.).
} 
Welche Auswirkungen hat die Entwicklung auf den Nationalstaat? Der Nationalstaat bekommt, so die Auffassung der Regulationstheoretiker, die durch ihn anfangs mit geförderten wirtschaftlichen Kräfte nicht mehr in den Griff. Er vermag immer weniger die Funktionen einer Regulation des Kapitalismus wahrzunehmen (vgl. Röttger 2003: 29). Zwar führe die Internationalisierung der Finanzmärkte und die Internationalisierung der Arbeitsteilung nicht zu einem Verschwinden der Nationalstaaten (vgl. Aglietta 2000: 42), aber es setze zwischen den Nationalstaaten immer mehr ein „sozialer Unterbietungswettstreit" ein. Da das Kapital zunehmend global agiere, die Arbeitskräfte aber weiterhin stark nationalstaatlich ausgerichtet seien, konzentriere sich der „nationale Wettbewerbsstaat“ darauf, „in Konkurrenz mit anderen Staaten günstige Verwertungsvoraussetzungen zu schaffen“. Unter Labeln wie der ,Deutschland AG' begriffen sich, so beispielsweise die Diagnose von Joachim Hirsch, die Nationen selbst als kapitalistische Unternehmen, die "glatt und effizient funktionierend“ auf ein "ökonomisches Ziel ausgerichtet" seien (Hirsch 1995: 103 ff.; siehe auch Hirsch 2005: 15 ff.). Unter diesen Bedingungen falle es, so die Einschätzung der Regulationstheorie, zunehmend schwer, den fordistischen Lohnkompromiss aufrechtzuerhalten.

Die Stärke des Regulationsansatzes ist, dass er ein Gerüst geliefert hat, mit dem die groben Linien der ökonomischen Veränderung sowohl theoretisch als auch empirisch erfasst werden konnten. Über die Debatte, ob wir es mit einem Fordismus, Hochfordismus, Neofordismus oder Postfordismus zu tun haben, verlor die marxistische Wirtschaftssoziologie die gesamtwirtschaftlichen Entwicklungen nicht aus dem Blickfeld und trug dazu bei, dass dieser Strang der Soziologie nicht zu einer reinen Betriebs- oder Arbeitssoziologie verkümmerte. Durch das marxistische Grundkonzept wirkt die Regulationstheorie geschlossener und stringenter als die eher empirisch orientierten Ansätze der Neokorporatismus-Forschung (vgl. Scharpf 1992; 1989), der vergleichenden Wohlfahrtsstaatsforschung (vgl. Esping-Andersen 1990) und der Varities-of-Capitalism-Ansätze (vgl. Hall, Soskice 2001; 2003; Hall, Gingerich 2004). ${ }^{10}$ Die Prämissen dieser regulationstheoretischen Herangehensweise dürfen nicht übersehen werden: Mit den Diagnosen des Spätkapitalismus, des Neokapitalismus, des Fordismus oder des Postfordismus wird darauf abgehoben, dass die Marx'sche Analyse nach wie vor gültig sei und im Vergleich mit den zur Lebenszeit von Marx herrschenden Formen der ökonomischen Produktion lediglich graduelle Unterschiede bestünden. Die Interventionen des ,Wohlfahrtsstaates', die Marx so nicht voraussagen konnte, hätten zwar zu einem ,Stillsetzen' des Klassengegensatzes geführt, aber die Klassengegensätze konnten, so jedenfalls die Annahme, in einer Krise jederzeit wieder ausbrechen. Damit grenzte man sich von den Labeln der Wohlfahrts-, Dienstleistungs- oder später auch der Wissensgesellschaft ab, die behaupten, dass die Gesellschaft "so durch und durch von der ungeahnt entfalteten Technik bestimmt ist“, dass demgegenüber das „soziale Verhältnis, das einmal den Kapitalismus definierte“, also die Ausbildung der Lohnarbeitsverhältnisse und die Entstehung der Klassengegensätze, an Relevanz eingebüßt hat (vgl. Adorno 1969: 12; Beckenbach 1991: 94 f.).

\footnotetext{
${ }^{10}$ Wissenschaftssoziologisch wäre es interessant zu überprüfen, ob es sich bei der Neokorporatismus-Forschung, der vergleichenden Wohlfahrtsstaatsforschung und den Varieties-of-Capitalism-Ansätzen nicht einfach nur um an der Oberfläche ,entmarxisierte Ansätze' handelt, die alle ein mehr oder minder verstecktes Primat der Ökonomie erhalten haben. Es fehlt meines Erachtens bisher weitgehend eine Gegenüberstellung dieser Ansätze mit der Regulationstheorie. Siehe aber die kritische Auseinandersetzung von Boyer 2005.
} 


\section{Dependenztheorie und Weltsystemtheorie}

Schon Karl Marx versuchte in seiner Analyse des Kapitalismus den Welthandel mit einzubeziehen. Die „Entdeckung der Gold- und Silberländer in Amerika“, „die Ausrottung, Versklavung und Vergrabung der eingeborenen Bevölkerung in die Bergwerke“, die „beginnende Ausplünderung von Ostindien“, die "Verwandlung von Afrika in ein Gehege zur Handelsjagd auf Schwarzhäute“ sei die „Morgenröte der kapitalistischen Produktionsära gewesen“. Der außerhalb Europas direkt durch „Plünderung, Versklavung und Raubmord erbeutete Schatz" sei direkt in das „Mutterland“ zurückgeflossen und hätte sich dort in Kapital verwandelt (Marx 1962 [1867]: 779, 781). Die Dependenztheorie und die Weltsystemtheorie entwickeln diese auf globale Wirtschaftsprozesse gerichtete Perspektive weiter und versuchen daraus eine umfassende Gesellschaftstheorie zu entwickeln. Die Dependenztheorie und die Weltsystemtheorie werden dabei häufig als zwei unterschiedliche Theorien präsentiert. Sie haben jedoch so viele Gemeinsamkeiten, dass die Darstellung als zwei unterschiedliche Theorien eher aus innerwissenschaftlichen Abgrenzungsbestrebungen denn aus Theoriekontroversen heraus erklärt werden kann (vgl. sehr früh zu den Ähnlichkeiten Brewer 1980; Chirot, Hall 1982). ${ }^{11}$ Folglich findet sich hier eine kombinierte Darstellung der beiden Theorien und nur vereinzelt wird auf Differenzen dieser beiden Theorien eingegangen. ${ }^{12}$

Die Hauptaussage der Dependenztheorie und die der Weltsystemtheorie sind einfach zusammenzufassen. Die Theorien erklären die ,Unterentwicklung' von Kolonien und später den Dritte-Welt-Ländern mit den Wirtschaftsbeziehungen zwischen dem entwickelten ,Zentrum' (bei den Dependenztheoretikern ,Metropolen') und der weniger fortgeschrittenen ,Peripherie'. Die Zentren sind technisch hoch entwickelt und bieten Fertigprodukte und Industriegüter an, während die Peripherie als Lieferant für Rohstoffe und landwirtschaftliche Güter dient. Bei diesem Tausch wird die Peripherie gegenüber den Zentren benachteiligt. Die Handelsbeziehungen laufen dabei über Vermittlungsinstanzen, die in der Dependenztheorie als Satelliten (nationale oder lokale Metropolen) (vgl. Frank 2000: $161 \mathrm{f}$.) und in der Weltsystemtheorie als Semiperipherien (vgl. Wallerstein 1974b: 125 ff.) bezeichnet werden. Diese Vermittlungsinstanzen bestehen entweder aus Regionen, die ökonomisch abgestiegen sind, oder aus Regionen, die sich aus der Peripherie absetzen konnten. Die Funktion dieser Vermittlungsinstanzen wird darin gesehen, die Krisenanfälligkeit des Systems zu reduzieren. Eine Mittelinstanz führe dazu, dass sich eine ausgebeutete Gruppe nicht einfach erheben kann und die Herrschaftsverhältnisse umkehrt (vgl. dazu Wallerstein 1974a; 1991). ${ }^{13}$ Die Ungleichheit zwischen Zentrum und Peripherie kann sich dabei zunehmend verschärfen. Die Staaten in der Peripherie sind gezwungen, eine große Menge an Billigprodukten zu verkaufen, um sich wenigstens einige teure Produkte aus dem Zentrum leisten zu können,

\footnotetext{
${ }^{11}$ Es wäre interessant zu untersuchen, ob die Erklärungen des Dependenztheoretikers André Gunder Frank nicht der Weltsystemtheorie von Immanuel Wallerstein stärker ähneln als den Erklärungen anderer Dependenztheoretiker wie Fernando H. Cardoso oder Enzo Faletto.

${ }^{12}$ Die besten deutschsprachigen Auseinandersetzungen mit neueren Ansätzen der Weltsystemtheorie sind Heigl 2005 und Hack 2005.

${ }^{13}$ Das Argument ist ganz ähnlich wie in der neueren marxistischen Klassentheorie. Siehe dazu den folgenden Abschnitt 4 und da besonders die Luhmann'sche Auseinandersetzung mit dem Marx'schen Klassenkonzept.
} 
die dort unter hohen Lohnkosten hergestellt werden. Weil in der Peripherie niedrige Löhne gezahlt werden, gibt es nach Auffassung der Dependenz- und Weltsystemtheoretiker keinen Druck, technische Innovationen zu entwickeln, um den Lohnkostenanteil zu drücken. Dagegen würden im Zentrum der Druck der Gewerkschaften, die wachsende Qualifikation der Arbeitnehmer und branchenspezifische Oligopole dazu führen, dass die Löhne hoch bleiben. Durch diesen Prozess wird die Kluft zwischen der Peripherie mit ihren niedrigen Löhnen und dem Zentrum mit den hohen Löhnen noch größer. Die Terms of Trade zwischen dem Zentrum und der Peripherie verschlechtern sich weiter (vgl. für die Dependenztheoretiker Prebisch 1959; 1962; für die Weltsystemtheoretiker Hopkins, Wallerstein 1982: $44 \mathrm{ff}$.).

Eine Stärke der Weltsystemtheorie und mit Abstrichen auch der Dependenztheorie ist, dass sich aus ihrer ökonomischen Analyse der Weltwirtschaft eine Theorie des Staates ableiten lässt. Die Unterschiede zwischen Zentrum und Peripherie sind ökonomisch begründet, werden dann aber politisch abgesichert. Weil die Staaten, die dem Zentrum näher sind, über mehr Ressourcen verfügen, können sie eine auch politische Herrschaft über die Staaten der Peripherie erreichen. Diese Herrschaft kann über so unterschiedliche Wege wie direkte Kolonialisierung, Einrichtung von Marionettenregierungen oder über internationale Organisationen erfolgen. Die politische Macht kann dann wiederum dazu genutzt werden, um den ungleichen ökonomischen Austausch weiter zu forcieren (vgl. Hopkins, Wallerstein 1982: 511; Wallerstein 1984b: 46 ff.). Sowohl die Dependenztheorie als auch die Weltsystemtheorie sehen vor, dass sich die Zentren auch verschieben können. Besonders interessant sind dabei die Veränderungen bei den Hegemonialmächten, die sich jeweils nach militärischen Niederlagen wichtiger Konkurrenten ausgebildet haben. In der Mitte des siebzehnten Jahrhunderts waren besonders die Niederlande eine solche Hegemonialmacht. Diese Rolle übernahm dann in der Mitte des neunzehnten Jahrhunderts Großbritannien und in der Mitte des zwanzigsten Jahrhunderts die USA (vgl. Wallerstein 1984a). Dabei gehen sowohl die prominentesten Vertreter der Dependenztheorie (vgl. Frank 1998) als auch der Weltsystemtheorie (vgl. Wallerstein 2003) in Abgrenzung von einer Reihe von Imperialismustheoretikern davon aus, dass sich das Zentrum zunehmend von Nordamerika nach Asien verlagern wird. Die Frage sei nicht mehr, so Wallerstein, ob die US-Hegemonie erodieren wird, sondern nur noch, ob die USA den Weg eines ehrenvollen Abgangs finden (vgl. Wallerstein 2003).

Sowohl die Dependenztheorie als auch die Weltsystemtheorie können als Gegenentwurf zu der in den fünfziger und sechziger Jahren dominierenden Modernisierungstheorie begriffen werden. So stellte Immanuel Wallerstein (1976: 131) seine weltsystemtheoretisch ausgerichtete Kritik an der Modernisierungstheorie auf dem Kongress der US-amerikanischen soziologischen Gesellschaften unter den Titel ,Ruhe in Frieden' und behauptete, dass Dependenztheorie und Weltsystemtheorie der Modernisierungstheorie eine Beerdigung erster Klasse beschert hätten (vgl. auch Swedberg 1987: 94). Die Dependenz- und Weltsystemtheorie drehen die Perspektive der Modernisierungstheorie um 180 Grad. Sie stellen die These auf, dass Probleme der Peripherie nicht wie die Modernisierungstheoretiker behaupten auf einen zu geringen Grad von Modernität zurückgeführt werden können, sondern auf einen seit mehreren Jahrhunderten andauernden Ausbeutungsprozess durch die Industrieländer. Die Unterentwicklung ist damit nicht das Ergebnis von in den traditionalen Gesellschaften angelegten ,Modernisierungsbremsen', sondern der häufig gewaltsa- 
men Einbindung der Peripherie in die internationale Arbeitsteilung (vgl. ECLA 1970). Die Provokation der Weltsystemtheorie besteht darin, dass die Globalisierung nicht wie bei vielen aktuellen Zeitdiagnostikern als ein aktuelles Phänomen bezeichnet wird. Vielmehr geht Wallerstein davon aus, dass durch den Handel mit Waren über nationale Grenzen hinweg die Konturen des Weltsystems bereits während des sogenannten langen sechzehnten Jahrhunderts in Europa entstanden sind. Seit dieser Zeit werden Waren im Rahmen einer kapitalistischen Weltökonomie über nationale Grenzen hinweg verschoben. Spätestens zu Beginn des neunzehnten Jahrhunderts sei das Weltsystem vollständig ausgebildet gewesen. Im Rahmen dieses über 400 Jahre ablaufenden Prozesses hat es nach Auffassung von Wallerstein eine Reihe von ,säkularen Trends' gegeben, die zu einer immer weiteren Konsolidierung und Absicherung des Weltsystems geführt haben. Das Weltsystem sei in dieser Zeit in die letzten Ecken dieser Welt expandiert und hätte beispielsweise dazu geführt, dass die Subsistenzwirtschaft zunehmend durch kapitalintensive verkaufsorientierte Landwirtschaft ersetzt wurde. Begleitet wurde dieser Expansionstrend durch den Trend einer weitgehenden Kommodifizierung: immer mehr Ressourcen wurden zu mit einem Tauschwert ausgestatteten Waren und dadurch durch den Handel im Weltsystem erfasst (vgl. Hopkins, Wallerstein 1982: 53 ff.).

Sowohl die Dependenztheorie als auch die Weltsystemtheorie haben unter Marxisten teilweise heftige Kritik hervorgerufen. Eine erste marxistische Kritiklinie bemängelt, dass die Analyse von Dependenztheoretikern wie André Gunder Frank, aber auch von Weltsystemtheoretikern wie Immanuel Wallerstein und Terence K. Hopkins vorrangig an der Zirkulationsebene des Warenverkehrs ansetze, die Produktionsverhältnisse besonders in der Peripherie jedoch aus dem Blickfeld verliere (vgl. dazu beispielsweise Brenner 1976). Der expandierende Kapitalismus müsse letztlich in den Satelliten, so besonders das Argument von Ernesto Laclau (1971), nicht die feudalistischen durch kapitalistische Strukturen ersetzen, um Ausbeutungsverhältnisse zu generieren. Häufig wurden gerade in den Satelliten wie beispielsweise im Fall der Sklaverei - vorkapitalistische Produktionsweisen überhaupt erst eingeführt, um entsprechend Profite aus den Ländern herauszuziehen. Nicht jede Warenproduktion für den Weltmarkt sei, so Laclau, notgedrungen kapitalistisch. Erst wenn die Arbeitskräfte im oben dargestellten Marx'schen Sinne doppelt frei seien - persönlich frei und frei von Produktionsmitteln -, könne überhaupt erst von einer kapitalistischen Produktionsweise geredet werden. ${ }^{14}$

Eine zweite auf Marx basierende Richtung kritisiert die Vernachlässigung der Analyse von Klassenverhältnissen in der Dependenztheorie und in der Weltsystemtheorie. Die nur rudimentär ausgearbeitete Marx'sche Klassenanalyse basiert letztlich auf die Bestimmung der Produktionsverhältnisse. Die Klasse der Kapitalbesitzer (sei sie auch noch so arm) stehe der Klasse der Arbeiter gegenüber, die frei von Produktionsmitteln ist. Sowohl in der Dependenztheorie als auch in der Weltsystemtheorie wird die Differenz zwischen den Klassen tendenziell abgelöst durch die geographisch bestimmten Differenzen zwischen Zentrum und Peripherie. Damit, so jedenfalls die Kritik, würde der Blick für die Klassengegensätze

\footnotetext{
${ }^{14}$ Die Kritik richtete sich vor allem gegen diejenigen Dependenztheoretiker, die Unterentwicklung ausschließlich mit ungleichem Tausch erklären, sie lässt sich mit Abstrichen aber auch gegen die Weltsystemtheorie vorbringen.
} 
sowohl in den Industrie- als auch in den Entwicklungsländern aus dem Blickfeld verschwinden.

In einer dritten Kritiklinie wird aus einer marxistischen Perspektive argumentiert, dass der Kapitalismus sich bisher noch gar nicht weit genug durchgesetzt hätte. Der Imperialismus sei nicht, wie von den meisten marxistischen Forschern angenommen, das letzte Stadium des Kapitalismus, sondern erst durch den Imperialismus käme es zur weltweiten Durchsetzung der kapitalistischen Produktionsverhältnisse. Grundlegender - und letztlich den marxistischen Rahmen verlassend - war die Kritik, die den Dependenztheoretikern einen ökonomischen Reduktionismus vorwirft (vgl. Booth 1985: 773). Der Vorwurf zielt auf die Vernachlässigung des politischen, rechtlichen, wissenschaftlichen und religiösen Eigenlebens (vgl. Beck 1997: 66 f.), wurde aber häufig durch den Vorwurf eines verkürzten Verständnisses von Kultur artikuliert. Gerade in den empirischen Studien sowohl der Dependenztheoretiker als auch der Weltsystemtheoretiker erscheine, so der Vorwurf, Kultur als eine "Verkleidung von politisch-ökonomischen Interessen“ (Hack 2005: 148), bestenfalls als ein „ideologisches Schlachtfeld des ökonomischen Weltsystems“ (Münch 2004: 324). Die Herausforderung für die Dependenztheorie und Weltsystemtheorie besteht darin, ihre politikökonomischen Prämissen sowohl theoretisch als auch empirisch gegen die seit den siebziger Jahren entstandenen Ansätze der Weltgesellschaft zu verteidigen. Besonders mit den Konzepten von Peter Heintz (1982), Niklas Luhmann (1971) und John Meyer (1980) liegen inzwischen Konzepte vor, die versuchen verschiedene Komponenten wie Wirtschaft, Wissenschaft, Religion und Politik gleichrangig zu behandeln.

\section{Klassentheorien}

Die Marx'sche Klassentheorie ist für die Wirtschaftssoziologie deswegen relevant, weil hier - im Gegensatz beispielsweise zu systemtheoretischen Klassen- und Schichtungsansätzen (vgl. Luhmann 1985; Kieserling 2006) - die Klasse über den Besitz an Produktionsmitteln bestimmt wird. Gleichzeitig stellte die Marx'sche Klassentheorie aber einen (häufig sogar den) Ansatz dar, mit dem der Schritt von einer Wirtschafts- zu einer Gesellschaftstheorie gegangen werden konnte. Aus der Verortung in den beiden wirtschaftlich bestimmten Klassen konnten - in einer simplifizierten Form - Positionen in Politik, Recht, Wissenschaft oder Religion abgeleitet werden. Die Herausforderung für die Marx'sche Theorieentwicklung bestand und besteht darin, dass sich zwei Klassenbegriffe gegenüberstehen, die sich nicht ohne weiteres miteinander kombinieren lassen. Bei dem Verständnis der ,Klasse an sich' wird Klasse als eine analytische Einheit verstanden. Die Klasse erscheint nur auf dem „Radar der proletarischen Avantgarde“ oder auf dem „Bildschirm der Wissenschaft“ (Luhmann 1985: 128). Sie scheint deswegen ,objektiv' vorhanden, was aber für die Wissenschaftler oder die proletarische Avantgarde nicht heißen muss, dass auch die Subjekte (gemeint sind die Proletarier) ihre Klassenlage erkennen. Die Klassenlage kann sich also nur einem politischen Aktivisten mit tiefen Einblicken in die Verhältnisse oder einem Wissenschaftler mit der Fähigkeit zu detaillierten Messungen der Sozialstruktur erschließen. Im unwahrscheinlichen Fall, dass Erika Mustermann oder Otto Normalverbraucher die wissenschaftlichen oder agitatorischen Interpretationen von einer ,verschärften Klassengesellschaft', einer ,Klassen- 
gesellschaft ohne Klassen' oder einer ,klassenlosen Gesellschaft' in die Hand bekommen, können sie als Subjekte vielleicht gar nicht so viel mit ihrer vermeintlich objektiven Verortung in der Klassenstruktur anfangen.

Mit dem Diktum der ,Klasse für sich' wird proklamiert, dass diejenigen, die sich in ähnlichen Klassenlagen befinden, dies schon bemerken würden, besonders dann, wenn es darum gehe, diese Lage grundlegend zu verändern. Die Klasse ist also nicht mehr nur objektiv vorhanden, sondern erschließt sich auch den Subjekten. Die ,objektive Klassenlage' führt, so die Annahme, letztlich zur Ausbildung vergleichbarer und ähnlicher Lebenserfahrungen und dann auch zu einem Bewusstsein der eigenen Klassenlage. Erika Mustermann und Otto Normalverbraucher werden sich ihres Status als Arbeiter, ihrer Klassenlage bewusst und entwickeln eine vergleichbare Bewusstseinslage (vgl. Marx 1959 [1847]: 180; siehe auch Kocka 1983: 26; Luhmann 1985: 128). Aus dieser Differenz zwischen ,Klasse an sich' und ,Klasse für sich', aus der Unterscheidung zwischen ,objektiver Klassenlage' und ,subjektiver Verarbeitung' kann man ein umfangreiches Forschungsprogramm generieren: Wie erklärt man aus einer marxistischen Perspektive, dass sich empirisch, jedenfalls in den entwickelten Industriestaaten, kaum eine ,Klasse für sich' erkennen lässt und die Arbeiter nur beschränkt ein politisches Bewusstsein als Arbeiter ausbilden? Weswegen wird aus der ,Klasse an sich' nicht oder nur begrenzt eine ,Klasse für sich'? Wie kommt es, so die alltagssprachliche Formulierung der Frage, dass man nicht allein schon anhand der Lebensführung darauf schließen kann, ob jemand ein Proletarier ist? Die intellektuelle Herausforderung für die an Klassenfragen interessierte Wirtschaftssoziologie bestand darin, dass spätestens seit dem Zweiten Weltkrieg in der Soziologie zunehmend Zweifel laut wurden, ob die von Marx propagierten Klassenbildungsprozesse zu einer Analyse der Gesellschaft taugten. Mit Begriffen wie der „Klassengesellschaft im Schmelztiegel“ (Geiger 1949), der „nivellierten Mittelstandsgesellschaft" (Schelsky 1965), der "klassenlosen Einheitsgesellschaft" (Landshut 1956), der „Auflösung proletarischer Milieus“ (Mooser 1983) oder der „Pluralisierung von Lebenslagen und der Individualisierung von Lebensläufen“ (Beck 1986) verwiesen Sozialwissenschaftler darauf, dass sich neue Schichtungslinien in der Gesellschaft ausbildeten, die weit wichtiger waren als die einende Kraft der Produktionsverhältnisse. Als Reaktion auf diese als ,bürgerliche Soziologie' verschrienen Erklärungsrichtungen wurden Modifikationen der marxistischen Klassentheorie vorgenommen. Es bildeten sich letztlich zwei Ansätze aus, die auch für die Wirtschaftssoziologie relevant sind. ${ }^{15}$

Die Vertreter des in der Regel durch Marx inspirierten Klassendifferenzierungsansatzes lehnen die Nivellierungsthesen der als ,bürgerlich' abgetanen Soziologie ab, greifen aber die schon von Marx und Engels angedeutete Möglichkeit der Bildung von intermediären Klassenpositionen und von Binnendifferenzierungen sowohl der Kapitalistenklasse als auch der Arbeiterklasse auf. Im Mittelpunkt des Klassendifferenzierungsansatzes steht dabei die Frage, ob der technische Wandel in der industriellen Produktion nicht zu einer so weitgehenden Differenzierung der Klasse geführt hat, dass kaum noch von einer kollektiven Erfahrungsbasis oder gar einer kollektiven Bewusstseinslage ausgegangen werden kann. Der

\footnotetext{
${ }^{15}$ Berger (1999) stellt zu Recht fest, dass es die Klassentheorie in Form eines festgelegten Kanons abgrenzbarer Lehrmeinung nicht gibt. Insofern ist auch die hier genutzte Unterscheidung in Klassendifferenzierungsansatz und Klassenvermischungsansatz lediglich als Ordnungsschema für die verschiedenen Autoren zu verstehen.
} 
französische Soziologe Serge Mallet, einer der prominentesten Vertreter dieses Ansatzes, stellt die Ausbildung einer ,neuen Arbeiterklasse' fest. Aufgrund der Entwicklung der Produktionsmittel werde von den Arbeitern nicht mehr vorrangig körperliche Arbeit verlangt, sondern technische Intelligenz, die Fähigkeit zur Kontrolle komplexer technischer Systeme und die Bereitschaft, Verantwortung für den Produktionsprozess zu übernehmen. Die ,neue Arbeiterklasse' bestände aus einem hoch spezialisierten Arbeitertyp, von dem ein hohes Bildungsniveau verlangt werde. Diese Klasse sei durch ein starkes berufliches und politisches Selbstbewusstsein geprägt. Ihr Drang nach einer stärkeren Kontrolle der Produktionsorganisation im Betrieb stoße jedoch auf die Herrschaftsansprüche des Managements kapitalistischer Unternehmen. Aus diesem Widerspruch bilde sich dann ein ausgeprägtes Klassenbewusstsein aus (vgl. Mallet 1969: 38 ff.; siehe auch Deppe 1971: 47). Der amerikanische Soziologe Erik Olin Wright geht beispielsweise davon aus, dass zwar der Produktionsmittelbesitz immer noch den Hauptunterschied in einer Klassengesellschaft ausmache, dass aber sowohl die Klassenlage der Produktionsmittelbesitzer als auch die Klassenlage der Lohnabhängigen weiter differenziert werden müsse. Genauso wie bei den Besitzern von Produktionsmitteln die Bourgeoisie von den kleinen Arbeitergebern und dem Kleinbürgertum unterschieden werden müsse, müssten beispielsweise bei den Lohnabhängigen Manager, Aufsichtspersonen und Arbeiter differenziert werden, weil sie in einem ganz unterschiedlichen Maße auf die Ressourcen der sie beschäftigenden Organisation zurückgreifen könnten. Innerhalb der Gruppe der Manager, Aufsichtspersonen und Arbeiter müssten dann noch deutliche Unterschiede in Bezug auf die Qualifikation gemacht werden (vgl. Wright 1985; für das Vorläufermodell Wright 1978; siehe auch Koch 1994: 67 ff.).

Die sich im Zuge des Klassendifferenzierungsansatzes ausbildende Debatte ist übersichtlich geblieben. Da die Frage, ob das ,verbürgerlichte Proletariat' ein Klassenbewusstsein hat und ein Bewusstsein als revolutionäres Subjekt ausbilden kann (vgl. eher dafür z. B. Mallet 1969; eher dagegen Deppe 1971), kaum noch Aufmerksamkeit hervorruft, wird die Hauptdebatte zwischen Vertretern der These einer Re-Proletarisierung einerseits und den Beobachtern einer verstärkten Klassendifferenzierung andererseits geführt. Die Frage ist, ob wir es mit einem Trend zur Verelendung der Arbeiterschaft und damit zu einer "Wiederkehr der Proletarität“, einer „Reproletarisierung“ und einer „Reetablierung einer Arbeiterklasse“ mit entsprechendem Klassenbewusstsein zu tun haben (Roth 1994: 12 ff.), oder ob sich die Tendenz zur Ausbildung eines ,verbürgerlichten Proletariats' noch radikalisiert und durch eine fortschreitende Fragmentierung des Proletariats eine ,Klassengesellschaft ohne Klassen' entsteht.

Im Klassenvermischungsansatz wird argumentiert, dass sich von den Produktionsverhältnissen nicht ohne weiteres auf soziale Klassen schließen lasse. Im Begriff der ,Klasse für sich' wird, wie oben schon ausgeführt, davon ausgegangen, dass sich die Produktionsverhältnisse in realen sozialen Klassen wiederfinden lassen. Damit wird eine Vorstellung nahe gelegt, dass man entweder Kapitalist oder Arbeiter ist. Der Klassenvermischungsansatz schließt nicht aus, dass die Ausbildung von sozialen Klassen in einer kapitalistischen Wirtschaft möglich ist, in der Einzelpersonen Eigentümer von Unternehmen sind und die Arbeiter als Verkäufer ihrer Arbeitskraft über keine Möglichkeit zur Teilhabe am Kapital verfügen. Der Klassenvermischungsansatz bringt jedoch Zweifel vor, ob es die aktuellen Entwicklungen des Kapitalismus besonders im Finanzbereich der fortgeschrittenen europä- 
ischen, asiatischen und amerikanischen Industriestaaten ermöglichen, diese Trennung noch aufrechtzuerhalten. Schon Marx hat im dritten Band des Kapitals herausgearbeitet, dass sich aufgrund der zunehmenden Bedeutung von Aktiengesellschaften die Besitzrechte am kapitalistischen Unternehmen immer weniger an der Person des Einzelunternehmers festmachen lassen, sondern sich Konglomerate von Kapitalbesitzern ausbilden. Die in Aktiengesellschaften häufig zu findende breite Verteilung des Kapitaleigentums sei, so Marx, die „Aufhebung des Kapitals als Privateigentum innerhalb der Grenzen der kapitalistischen Produktionsweise selbst“" (Marx 1964 [1894]: 452).

Der für die Frage der Klassenbildung zentralere Effekt ist, dass aufgrund der Diffusion des Kapitalbesitzes das Verfügungsrecht des einzelnen Privatunternehmers immer mehr auf eine Gruppe von an den Produktionsmitteln ,eigentumslosen Führungskräften' übergeht. Schon als in den dreißiger Jahren erste Überlegungen zu einer ,Demokratisierung' des Finanzkapitals aufkamen, wiesen Adolf Berle und Gardiner Means darauf hin, dass eine Streuung des Aktienbesitzes auf eine Vielzahl von Personen nicht zu einer breiteren Kontrolle des Kapitals führe, sondern vielmehr die Stellung des Managementstärke. In den an der Börse notierten Unternehmen werde der Manager, eigentlich ein ,unselbständiger Angestellter', der ,unternehmerisch wirkende' Herrscher, wenn sich der Kapitalbesitz nicht mehr in der Hand von einem oder zwei zentralen Kapitaleignern, sondern in der Hand einer Vielzahl von Kapitalbesitzern befinde (vgl. Berle, Means 1934).

Die Quintessenz ist, dass aufgrund der Auflösung der Polarität von Kapitalist und Arbeiter als zwei sozial unterscheidbare Gruppen Klassenbildungsprozesse nicht mehr zu erwarten sind. Bei aller Stärke einer analytischen Unterscheidung zwischen Kapital und Arbeit eignet sich diese Differenz aus der Perspektive des Klassenvermischungsansatzes nicht für wissenschaftlich oder politisch begründete Hoffnungen auf revolutionäre Umwälzungen. Aber auch wenn der Klassendifferenzierungsansatz und der Klassenvermischungsansatz letztlich zu einer Aufgabe von Klassenkampfkonzeptionen führen, so ist doch wichtig herauszustellen, dass beide Ansätze an einer Bestimmung der Klassen über die Produktionsverhältnisse festhalten. Sie stehen damit letztlich in einer marxistischen Traditionslinie der Klassentheorie. Diese produktionistisch begründete Klassenanalyse gerät jedoch immer mehr von Seiten einer kulturalistischen Klassentheorie unter Druck. Die kulturalistischen Ansätze weisen auf die Gefahr einer reduktionistischen Verkürzung von Bewusstsein zum Derivat objektiver Verhältnisse hin. Die Arbeiter seien genauso wenig wie Kapitalisten Träger von Bewusstseinsformen, die sich aus einer schlichten Ableitung aus den ökonomischen Beziehungen ergeben. Vielmehr bildeten Menschen Deutungsmuster aus, mit denen sie versuchten, die von ihnen erfahrene Realität zu verarbeiten. Diese Deutungsmuster würden zwar durch die ökonomischen Verhältnisse beeinflusst, wären aber in ihren Ausprägungen relativ autonom. In ihrer entschiedensten Form steht Pierre Bourdieu für diese sich von den Produktionsverhältnissen lösende Klassenanalyse (vgl. den Beitrag von Fley sowie von Schimank und Volkmann in diesem Band). Die kulturalistischen Klassenansätze haben zur Konsequenz, dass die Verbindungen zwischen der Klassentheorie und der Wirtschaftssoziologie tendenziell gekappt werden. Bei Aufgabe der produktionistischen Bestimmung der Klassenzugehörigkeit macht es nur noch wenig Sinn, der Wirtschaftssoziologie einen bevorzugten Zugang zur Bestimmung von Klassenverhältnissen einzuräumen. Die Theorie der Klas- 
sen wird Teil einer allgemeinen Gesellschaftstheorie, ohne dass die Wirtschaftssoziologie noch einen prädestinierten Zugang für sich reklamieren kann.

\section{$5 \quad$ Labour Process Debate}

Die Labour Process Debate ist dadurch gekennzeichnet, dass sie den Schritt von einer Wirtschafts- zu einer Gesellschaftstheorie über die Bestimmung der Produktionsbedingungen in den Betrieben zu gehen sucht. Ausgangspunkt der Analyse sind die Strategien zur Aneignung des Mehrwertes. Jeder einzelne Kapitalist, so schon Marx, strebt danach, den Profit in seinem eigenen Betrieb zu erhöhen. Sein „Heißhunger“ nach Mehrwert ist nicht zu stillen (Marx 1962 [1867]: 249). Er versucht deswegen nicht nur, möglichst viel Arbeit für sein Geld $\mathrm{zu}$ bekommen, sondern setzt auf technische, organisatorische und arbeitspsychologische Innovationen, die zu einer erhöhten Arbeitsproduktivität führen. Dem Kapitalisten kann es dadurch gelingen, gegenüber der Konkurrenz einen Vorsprung zu erreichen, weil der Arbeitsaufwand, den er in ein Produkt steckt, geringer ist als der durchschnittliche Arbeitsaufwand in seiner Branche. Er realisiert, so Marx, einen „Extramehrwert“. ${ }^{16}$ Es gibt nach der Marx'schen Betriebsanalyse zwei Strategien des Kapitals, um mehr aus dem Arbeiter herauszuholen: Die Ausdehnung der Arbeitszeit, ohne dass der Kapitalist dafür mehr bezahlen muss (Steigerung des absoluten Mehrwerts), und die effektivere Nutzung der eingekauften Arbeitszeit (Steigerung des relativen Mehrwerts). ${ }^{17}$ Diese beiden Strategien zur Steigerung des Mehrwertes stehen, das machte schon Marx deutlich, miteinander in Beziehungen. Wenn die Ausdehnung der Arbeitszeit aufgrund von gesetzlichen Arbeitsschutzbestimmungen oder von tariflichen Arbeitszeitregelungen nicht möglich ist, wird das Kapital den Intensitätsgrad der Arbeit erhöhen. Gleichzeitig zerstört aber eine zu hohe Arbeitsintensität bei zu langer Arbeitszeit die Arbeitskraft. Deswegen mache eine Steigerung des Intensitätsgrads der Arbeit eine Verkürzung der Arbeitszeit unvermeidlich (vgl. Marx 1962 [1867]: 440).

Aufgrund ihrer starken Verankerung in der marxistischen Tradition waren einerseits die Arbeitszeitstrategien des Kapitals und andererseits die Rationalisierungsmaßnahmen zur Steigerung der Arbeitsintensität die beiden Hauptthemen der am Betrieb interessierten Arbeits- und Industriesoziologie. Besonders das dreizehnte Kapitel des Kapitals, in dem der Wechsel von der Manufakturfertigung zur Industrieproduktion dargestellt wird, kann als die Marx'sche ,Urquelle' der Arbeits- und Industriesoziologie gesehen werden. Dieses Kapitel, so eine ketzerische Außenbetrachtung von Hans Paul Bahrdt, spiele für die Arbeits- und Industriesoziologie etwa die gleiche Rolle wie der Römerbrief für die protestantischen Theologen (vgl. Bahrdt 1982: 14). Auch wenn der Großteil der Wirtschafts-, Arbeits- und Indus-

\footnotetext{
${ }^{16}$ Aber dieser Extramehrwert verschwindet nach Auffassung von Marx, weil das ,Zwangsgesetz der Konkurrenz' die Mitbewerber dazu treibt, die Innovationen ebenfalls einzuführen. Somit verbreiten sich die zunächst isoliert eingeführten Maßnahmen allgemein (vgl. Marx 1962 [1867]: 336 ff.).

${ }^{17}$ Schon die Enquete Ouvrière von Marx zielte mit Fragen nach einer Verkürzung der Esszeiten, nach Überstunden zur Reinigung von Maschinen, nach Nachtarbeit oder nach saisonmäßig bedingter Überarbeit und mit Fragen nach einer Steigerung der Arbeitsintensivität auf diese beiden grundlegenden Strategien zur Mehrwertproduktion (vgl. Weiss 1936: 89).
} 
triesoziologen heutzutage auf Marxreferenzen verzichtet, kann man die Hauptdiskussionen innerhalb der auf Betriebe konzentrierten Forschungsrichtung als Auseinandersetzung mit den von Karl Marx herausgearbeiteten Profitmaximierungsstrategien verstehen.

Die naheliegende Herangehensweise der Arbeits- und Industriesoziologie war es, die Marx'schen Grundlagen zum Thema der Dequalifizierung fortzuschreiben. Für Marx setzte die Dequalifizierung mit dem Übergang von der Manufakturfertigung zur Industrieproduktion ein. Durch diese Umstellung verschob sich die Kontrolle über den Arbeitsprozess von den Arbeitern auf das Kapital. In den Manufakturen waren die Arbeiter ,formell' von den Produktionsmitteln getrennt gewesen. Schließlich waren die Kapitalisten und nicht die Arbeiter formell deren Besitzer gewesen. Aber im konkreten Arbeitsprozess hatten die Arbeiter aufgrund ihres Wissens über die Produktion große Gestaltungsmöglichkeiten gehabt. Der Kapitalist hatte lediglich die Werkzeuge und Materialien besessen, den Arbeitsprozess selbst aber nicht beherrscht. Marx nennt dies eine lediglich "formelle Subsumtion" der Arbeit unter das Kapital. Durch die Einführung der "großen Industrien“ wurden die Arbeiter auch der Kontrolle über den Arbeitsprozess beraubt. Der Arbeitsprozess wurde jetzt nicht mehr von den Arbeitern beherrscht, sondern vom Kapitalisten selbst oder von seinen Abgesandten. Die „formelle Subsumtion“ wurde ausgedehnt auf eine „reelle Subsumtion" der Arbeiter unter das Kapital (Marx 1962 [1867]: 532 ff.). Harry Braverman, der prominenteste Vertreter einer auf Marx sich berufenden Dequalifizierungsthese, argumentiert, dass die sich im zwanzigsten Jahrhundert zunehmend durchsetzenden Rationalisierungsstrategien dazu dienten, den Arbeitsprozess zunehmend von Erfahrungen, Kenntnissen und Traditionen des handwerklichen Könnens des Arbeiters zu trennen. Das Wissen, das die Arbeiter über Jahrzehnte und Jahrhunderte angesammelt hätten, werde systematisch auf das Management verlagert werden. Dies mache den Kapitalisten von den Qualifikationen des Arbeiters unabhängig und ermögliche es ihm, die Arbeitnehmerschaft ganz den Zielen, Vorstellungen und Plänen des Managements unterzuordnen. Der Kapitalist schlage, so die Grundaussage Bravermans, mit seinen Rationalisierungsstrategien zwei Fliegen mit einer Klappe: Erstens verfüge er über eine effiziente Organisationsstruktur, mit der sich der relative Mehrwert kontinuierlich steigern lasse, und zweitens ermögliche die Dequalifizierung der Arbeiter und die starke Zergliederung des Arbeitsprozesses eine bessere Kontrolle der Arbeiter (vgl. Braverman 1974: 124 ff.). Braverman schließt mit dieser Verbindung aus Rationalisierungsstrategien und Fragen der Kontrollstrategien des Managements direkt an eine bereits von Marx angerissene Spezifik des Arbeitsvertrages an. Während in einem simplen Kaufvertrag Leistung und Gegenleistung genau spezifiziert werden, kauft der Arbeitgeber mit einem Arbeitsvertrag Arbeitskraft nur in einer sehr abstrakten Form ein. Der Arbeitnehmer stellt mit dem Unterzeichnen eines Arbeitsvertrags eine Art ,Blankoscheck' aus und erklärt sich bereit, seine Arbeitskraft, seine Fähigkeit, seine Kreativität gemäß der ihm gestellten Aufgabe einzusetzen. Er verzichtet darauf, dass im Detail festgeschrieben wird, worin seine Leistungen im Einzelnen zu bestehen haben (vgl. Commons 1924: 284).

Der Deal zwischen Arbeitgebern und Arbeitnehmern ist so angelegt, dass der Arbeitnehmer sich den Unternehmenszielen unterwirft, den hierarchischen Anweisungen Gehorsam verspricht und dafür vom Arbeitgeber mit Geldzahlungen belohnt wird (vgl. Barnard 1938: 167 ff.). Bei den Arbeitnehmern entsteht eine folgenreiche ,Indifferenzzone', innerhalb derer sie zu den Befehlen, Aufforderungen, Anweisungen und Vorgaben der Vorgesetzten 
nicht Nein sagen können (vgl. Simon 1957). Der Vorteil für das Management des Unternehmens liegt auf der Hand: Die Mitarbeiter geloben eine Art Generalgehorsam gegenüber zunächst nicht weiter spezifizierten Befehlen und Weisungen. So ermöglichen sie dem Management, die Organisation sehr schnell und ohne umständliche interne Aushandlungsprozesse an veränderte Anforderungen anzupassen. Diesen Flexibilitätsvorteil erkauft sich der Kapitalist jedoch mit einem Kontrollproblem: Während der Arbeitsvertrag die Leistungen des Arbeitgebers (nämlich die Lohnzahlung) genau spezifiziert, sind die Gegenleistungen der Mitarbeiter nicht genau festgelegt. So kann der Arbeitnehmer versuchen, sich der Leistungserbringung so weit wie möglich zu entziehen. Der Einkauf von Arbeitskraft durch den Kapitalisten - die formelle Subsumtion des Arbeiters - ist deswegen nicht gleichbedeutend mit der realen Nutzung der Arbeitskraft durch das Kapital - die reelle Subsumtion (vgl. Marx 1962 [1867]: 532 f.). Wenn der Arbeitgeber also Arbeitskraft einkauft, kann er sich anders als bei den von ihm gekauften Gebäuden, Maschinen und Materialien - nicht sicher sein, dass sie sich reibungslos in den Arbeitsprozess einordnen lässt (vgl. Braverman 1974: 57; Friedman 1977: 78; Jürgens 1984: 61).

Die Transformation von Arbeitsvermögen, also was ein Arbeiter in der vom Kapitalisten gekauften Arbeitszeit leisten könnte, in Arbeitsleistung, also was er wirklich leistet, ist problematisch. In dieser Meinung stimmen eine marxistisch geprägte Arbeits- und Industriesoziologie mit der Institutionenökonomie überein. Erst durch die systematische Dequalifizierung der Arbeiter und ihre Unterwerfung unter ein tayloristisch-fordistisches Produktionsregime hätten, so die Aussage Bravermans in Anschluss an Marx, die Kapitalisten dieses ,Transformationsproblem' einigermaßen in den Griff bekommen (vgl. Braverman 1974: $124 \mathrm{ff}.)^{18}$

In der Auseinandersetzung mit Harry Braverman wurden dessen Thesen zur weiteren Dequalifizierung der Arbeiter in der Labour Process Debate besonders im anglo-amerikanischen Sprachraum stark modifiziert, wenn nicht sogar verworfen. Andrew Friedman stellt beispielsweise heraus, dass die ,direkte Kontrolle' mit einer stark reduzierten Verantwortung der Arbeiter und deren strenger Überwachung durch das Management nur eine Strategie zur Transformation von Arbeitsvermögen in Arbeit ist. Eine andere Möglichkeit besteht darin, über stabile Beschäftigungsverhältnisse die Arbeiter an den Betrieb zu binden und ihnen im Rahmen dieser Grundloyalität eine ,verantwortliche Autonomie' zuzugestehen (vgl. Friedman 1977).

Das Verdienst der Labour Process Debate ist gewesen, dass die Rationalisierungsprozesse unter einer genauen Beobachtung der Soziologie standen. In der Wissenschaft fand eine intensive Debatte über die Bewertung der beobachteten Rationalisierungsstrategien statt. Die hitzige Debatte, ob wir es mit einer postfordistisch-posttayloristischen Arbeitsor-

\footnotetext{
${ }^{18}$ Es ist in der Wissenschaft immer noch umstritten, wie verbreitet die tayloristisch-fordistische Produktionsweise in den Betrieben gewesen ist. Einige Wissenschaftler vertreten die Auffassung, dass das tayloristische und mit Abstrichen auch das fordistische Produktionsparadigma gerade in ihrer Zuspitzung im Begriff des ,Fordismus' eher ein Konstrukt der Wissenschaft denn hegemoniale betriebliche Realität gewesen sind. Besonders die Ideen Taylors scheinen in den USA Teil einer öffentlichen Effektivitätshysterie zu Beginn des zwanzigsten Jahrhunderts gewesen zu sein, die sich überall durchzusetzen schien außer in den Betrieben. Es gab für Hausfrauen Kurse zur wissenschaftlichen Haushaltsführung, in Kirchen wurden Komitees für kirchliche Effizienz begründet, die Universitäten und Schulen bezogen das wissenschaftliche Management mit ein, aber nur in ganz wenigen Betrieben wurde das ,Taylor-Prinzip' in seiner ganzen Breite umgesetzt (vgl. Haber 1964: 51 ff.; siehe auch Vahrenkamp 1976: 21 ff.; Moldaschl, Weber 1998: 347 ff.).
} 
ganisation oder mit einer Rückkehr des Fordismus-Taylorismus zu tun haben, waren letztlich Formeln, mit denen die Kontroverse geführt wurde, welche Rationalisierungsstrategien sich durchsetzen und welche Auswirkungen diese Strategien auf die Arbeitnehmer haben würden. Das Problem dieser Debattenstruktur war jedoch eine starke konzeptionelle ,Verengung' der durch Marx inspirierten soziologischen Diskussion (vgl. auch Kern 1997: 29). Letztlich lässt sich die Debatte in der Arbeits-, Wirtschafts- und Industriesoziologie, aber auch in großen Teilen der Betriebswirtschaftslehre, der Arbeitswissenschaft und der Organisationspsychologie in ein verhältnismäßig einfaches Ordnungsschema pressen. ${ }^{19}$ Interessant dabei ist, dass die Vertreter aller vier Ansätze letztlich ihren Ursprung in einer marxistisch geprägten Soziologie hatten.

Abbildung 1: Rationalisierungsdebatte in der Arbeits- und Industriesoziologie

\begin{tabular}{|l|l|l|}
\hline & $\begin{array}{l}\text { Stärker arbeitsteilige } \\
\text { Organisation als effiziente } \\
\text { Form der Wertschöpfung }\end{array}$ & $\begin{array}{l}\text { ganzheitliche Arbeitsformen } \\
\text { als effiziente Form der } \\
\text { Wertschöpfung }\end{array}$ \\
\hline $\begin{array}{l}\text { Herausstellen der Vorteile für } \\
\text { die Arbeiter }\end{array}$ & $\begin{array}{l}\text { Tendenz findet sich bei } \\
\text { Springer; geht auf Taylor und } \\
\text { Ford zurück }\end{array}$ & $\begin{array}{l}\text { Tendenz findet sich } \\
\text { beispielsweise bei Kern und } \\
\text { Schumann }\end{array}$ \\
\hline $\begin{array}{l}\text { Herausstellen der Nachteile für } \\
\text { die Arbeiter }\end{array}$ & $\begin{array}{l}\text { Tendenz findet sich bei } \\
\text { Braverman; Argumentation } \\
\text { geht letztlich auf Marx zurück }\end{array}$ & $\begin{array}{l}\text { Tendenz findet sich } \\
\text { beispielsweise bei Moldaschl }\end{array}$ \\
\hline
\end{tabular}

In der einen Hinsicht dreht sich die Debatte um die Frage, welche Arbeitsstruktur am ehesten zur Profitmaximierung beiträgt - eine stark arbeitsteilige, retaylorisierte Arbeitsstruktur (vgl. Springer 1999) oder eine eher auf ganzheitlichen Arbeitsformen basierende (vgl. Kern, Schumann 1984). In der anderen Hinsicht geht es um die Frage, wie sich die für das Kapital als rational darstellenden arbeitsteiligen oder ganzheitlichen Arbeitsformen für die Mitarbeiter auswirken: Tragen die postbürokratischen Strategien zur Zufriedenheit, Selbstverwirklichung, Befreiung bei oder nicht?

Eine Gemeinsamkeit in der Debatte ist auffällig: Egal, ob man einen Trend eher zur weiteren Taylorisierung oder eher zur Ausbildung ganzheitlicher Arbeitsformen beobachtete, es wurde immer davon ausgegangen, dass es bei den Strategien des Kapitals um die Steigerung der ökonomischen Rationalität des Unternehmens ging. Der Industriebetrieb erschien ganz in der Tradition von Marx als Produkt des modernen Kapitalismus, in dem die Profitorientierung und das Rentabilitätsprinzip als ,ultima ratio' herrschten (vgl. Lutz, Schmidt 1977: 171). Es dominierte nach wie vor ein der Marx'schen Tradition geschuldeter „heimlicher Effizienzdeterminismus“ (Ortmann 1994: 94). Diese Form der Herangehensweise, in der die gesamte Unternehmung vom Prinzip der Profitorientierung aus rekonstruiert wird, lässt sich in Anschluss an Max Weber als zweckrationales Organisationsverständnis

\footnotetext{
${ }^{19}$ Man möge mir verzeihen, dass ich hier in die ,Magie der Kreuztabelle' verfalle. In diesem Artikel verwende ich die Kreuztabelle lediglich als didaktische Heuristik, um theoretische Ansätze zu ordnen, nicht um im Parsons'schen Sinne Aussagen über soziale Sachverhalte zu treffen.
} 
bezeichnen. Es handelt derjenige zweckrational, so Weber, der in seinem Handeln verschiedene Zwecke gegeneinander abwägt, die günstigsten Mittel zur Erreichung der definierten Zwecke wählt und in diesem Auswahlprozess von Zwecken und Mitteln mögliche unerwünschte Nebenfolgen mit in Betracht zieht. Um Webers Kriterien der Zweckrationalität zu erfüllen, müssen sich die Entscheider über ihre Interessen, Wünsche und Werte im Klaren sein. Der Kapitalist muss sich beispielsweise des Ziels der Verwandlung von , $G^{\prime}$ in , $G^{\prime \prime}$ bewusst sein. Er muss möglichst vollständige Informationen über alle Handlungsalternativen sammeln und die Konsequenzen der Alternativen abwägen (vgl. Weber 1972 [1922]: 13).

Insgesamt kamen in der Soziologie immer mehr Zweifel auf, ob sich die Verhältnisse in den Betrieben aus den gesamtgesellschaftlichen Verhältnissen von Kapital und Arbeit erschließen lassen oder umgekehrt dann auch die gesellschaftlichen Verhältnisse aus den Verhältnissen in den Betrieben abgeleitet werden können. Der ,marxistische Funktionalismus', in dem die Funktionsweise aus den allgemeinen Bewegungsgesetzen des Kapitalismus abgeleitet wurde, geriet zunehmend in die Kritik (vgl. Littler 1990: 46 ff.). Das Eigenleben der Organisation hat die marxistische Arbeits-, Industrie- und Wirtschaftssoziologie genauso wenig in den Griff bekommen wie das Verhältnis von Gesellschaft und Organisation. Die theoretisch ambitionierten Ansätze wie zum Beispiel die Überlegungen von Burawoy (1979) im Rahmen der Arbeitsprozess-Debatte verharrten letztlich im objektivistischen Bezugsrahmen der Kritik der politischen Ökonomie und leiteten die, wenn auch gebrochenen Handlungslogiken in Betrieben aus den kapitalistischen Konkurrenzverhältnissen ab.

\section{Resümee: Theorie der Ausdifferenzierung des Wirtschaftssystems}

Der Marxismus bietet offensichtlich einen Schutz gegen eine $\mathrm{zu}$ schnelle Orientierung an wissenschaftlichen Moden, an kleingliedrigen Forschungsansätzen oder an zu offensiven interdisziplinären Brückenschlägen. Die Orientierung an Marx scheint die typischen Vorteile einer Orientierung an einer soziologischen Großtheorie mitzubringen. Zwingt diese doch dazu, auch Fragestellen mittlerer Reichweite auf eine ausgearbeitete Großtheorie zu beziehen. So scheint die Lektüre der Marx'schen politischen Ökonomie beispielsweise ein guter Schutz dagegen gewesen zu sein, an der unter dem Stichwort ,Globalisierung' laufenden Neuigkeitsdramatisierung mitzumachen. Statt das Phänomen der Globalisierung wie bei einigen Globalisierungsforschern erst nach dem Zweiten Weltkrieg wirkmächtig werden zu lassen, wurde gerade aus einer neomarxistischen Perspektive überzeugend herausgearbeitet, dass Handels-, aber auch Produktionsprozesse schon weit länger grenzüberschreitend ausgelegt gewesen sind. ${ }^{20}$ Auch als sich um die Jahrhundertwende einige Arbeits-, Industrie- und Wirtschaftssoziologen vom Hype um die New Economy anstecken ließen und (sehr vorschnell) bisher ungeahnte Rationalisierungsvorteile der New Economy vermuteten, war es die Lektüre der Teile über den Finanzmarkt im Dritten Band des Kapitals, die Neomarxisten davon abhielt, die Schimäre einer durch Informations- und Kommunikationstechnologie getriebenen Effizienzsteigerung mitzugehen. Der Profit erschien gerade aus dieser Perspek-

${ }^{20}$ Das Manko ist jedoch, dass beispielsweise politische Globalisierungsprozesse nicht ähnlich gut erklärt werden können. Hier scheinen die empirischen Ansätze aus dem Neoinstitutionalismus stärker zu sein. 
tive als ein Mythos, dem sich Unternehmen bedienten, um entsprechende Signale an den Kapitalmarkt zu senden (vgl. Kühl 2003). Aber kann man aus diesen überzeugenden Verwendungen des Marx'schen Theorieapparats auch darauf schließen, dass das gesellschaftstheoretische Projekt des Neomarxismus gelungen ist? Kann man der Eigensinnigkeit von gesellschaftlichen Teilbereichen wie Politik, Recht oder Wissenschaft einen relativ hohen Stellenwert beimessen und trotzdem immer noch die kapitalistischen Produktionsverhältnisse als Ausgangspunkt einer Analyse beibehalten?

Es fällt auf, dass bei der Kritik an der Regulationstheorie, an der Dependenztheorie, der Weltsystemtheorie, den marxistischen Klassentheorien und auch bei der Labour Process Debate immer wieder darauf verwiesen wird, dass die Bestimmung von Politik, Recht, Wissenschaft und Religion unterkomplex bleibt. So fällt beispielsweise bei den Regulationstheoretikern auf, dass man sich noch nicht auf eine allgemein geteilte Staatstheorie geeinigt hat, über die maßgeblich die Funktionsweise von Politik und Recht bestimmt werden kann. In der Weltsystemtheorie wird zwar ein Staatsverständnis erarbeitet, aber die Möglichkeiten, dass sich Staaten auch entkoppelt von Entwicklungen der Wirtschaft verändern können, bleiben unbeachtet. Im Rahmen der Labour Process Debate erscheint der Staat häufig nur als Feld der Auseinandersetzung zwischen unterschiedlichen, vorrangig über Positionen im Betrieb bestimmten Kräften.

Die marxistischen Ansätze in der Wirtschaftssoziologie ähneln überraschend den neoklassischen Ansätzen der Wirtschaftswissenschaften, von denen sie sich sonst so deutlich abgrenzen. Wie die marxistische Wirtschaftssoziologie tendieren auch die Wirtschaftswissenschaften dazu, die Gesellschaft mit der Wirtschaft gleichzusetzen. Der Wirtschaft wird tendenziell auch die "politische Funktion der Steigerung gesellschaftlicher Wohlfahrt", die „religiöse Funktion des Geldes als Gottesersatz“, die „pädagogische Funktion der Erziehung“ zugewiesen (Baecker 2006: 19), bloß dass diese Funktionen anders als bei den Neomarxisten tendenziell positiv hervorgehoben werden.

Wie könnte angesichts dieser Kritik ein alternativer Anschluss an Marx aussehen? In einer systemtheoretischen Lesart könnte man Beschreibungen wie das Interesse des Kapitalisten an der Vermehrung des Geldes des Geldes wegen oder den Wechsel von ,W-G-W' zu ,G-W-G' sehr wohl übernehmen. Wie die meisten Sozialwissenschaftler würden auch Systemtheoretiker mit Marx insofern übereinstimmen, dass das private Eigentum an Produktionsmitteln, der auf Expansion angelegte Erwerbsbetrieb, die abhängige Lohnarbeit als dominante Form der erwerbsorientierten Arbeitstätigkeit und die Verwandlung aller produzierten Güter und Dienstleistungen sowie der dafür notwendigen Arbeitskraft in marktfähige Waren zentrale Merkmale der kapitalistischen Wirtschaft sind (vgl. Weber 1972 [1922]; Aron 1964; Polanyi 1995). ${ }^{21}$

Systemtheoretisch gesprochen: Mit der Ausdifferenzierung der Wirtschaft als eigenständiges Funktionssystem zählt nur noch das ,Geld machen des Geldes wegen' (siehe G-WG). Man kann - anders als in vormodernen Gesellschaften - Geld heutzutage nicht mehr

\footnotetext{
${ }^{21}$ Bei Luhmann (1981: 397) wird die Ausdifferenzierung der Wirtschaft mit Hinweis unter anderem auf Polanyi 1978 (hier: Polanyi 1995) und Wallerstein (1974b) referiert. Türk (1987: 39) übersieht, dass es gerade der Clou der Luhmann'schen Argumentation ist, dass er zwar die Ausdifferenzierungsthese von Polanyi und von Wallerstein übernimmt, ohne damit aber deren These vom Primat der Ökonomie zu übernehmen. Eine ähnliche Argumentationsstrategie lässt sich auch bezüglich der Funktionssysteme Politik und Wissenschaft finden.
} 
dafür einsetzen, um Seelenheil, politische Ämter oder wissenschaftliches Renommee zu kaufen. Für Seelenheil muss man beten, für politische Ämter muss man bei Wahlen kandidieren und für wissenschaftliches Renommee von Fachkollegen akzeptierte Artikel publizieren. Geld kann man ,nur' dazu benutzen, um seine Konsumbedürfnisse zu befriedigen oder aus dem Geld noch mehr Geld zu machen (siehe G-W-G'). In der am weitesten gehenden Form der Ausdifferenzierung funktioniert die Wirtschaft nur noch nach einfachen Zweier-Schemata: In der Wirtschaft interessiert nur noch, ob gezahlt wird oder ob nicht gezahlt wird, und nicht, ob einem die Hautfarbe, das Geschlecht oder die politische Orientierung eines Kunden oder Verkäufers genehm ist. Der zentrale Unterschied ist jetzt jedoch, dass man die Ausdifferenzierung der Wirtschaft nicht mehr als das zentrale Merkmal der modernen Gesellschaft beschreibt. Vielmehr lassen sich parallel zu der von Karl Marx eindrucksvoll beschriebenen Ausdifferenzierung der Wirtschaft ähnliche Prozesse auch für gesellschaftliche Felder wie Politik, Wissenschaft oder Religion beschreiben. Die Ausdifferenzierung der Wirtschaft erscheint in dieser Analyse also nicht mehr als zentraler Sonderfall (Stichwort Primat der Ökonomie) der Ausdifferenzierung, sondern als einer von einigen wenigen prominenten Fälle der Ausdifferenzierung in der modernen Gesellschaft. ${ }^{22}$

Wenn man die verständliche marxistische Abwehrreaktion gegenüber der Systemtheorie außer Acht lässt, kann man die Theorie funktionaler Differenzierung sogar als eine Radikalisierung von Karl Marx lesen (vgl. auch Luhmann 1975a: 81; Luhmann 1997: 366). ${ }^{23}$ Die Eigensinnigkeit ausdifferenzierter gesellschaftlicher Teilbereiche wie Religion, Politik oder Liebe führt dazu, dass sich die Wirtschaft um vieles nicht mehr zu kümmern braucht. Erst unter diesen ,Entlastungsbedingungen' - und hier liegt die Radikalisierung - kann der Kapitalismus seine Dynamik entfalten (vgl. Luhmann 1997: 724 ff.).

Das systemtheoretische Stichwort dafür ist „legitime Indifferenz“ (Luhmann 1965: 35; Tyrell 1978: 183). Die Wirtschaft braucht in der modernen Gesellschaft keine Rücksicht darauf zu nehmen, dass die durch sie verursachte Massenarbeitslosigkeit zu einem Zusammenbruch des Renten- oder Gesundheitssystems führt. Wenn ein Unternehmer angesichts der von ihm durchgeführten Massenentlassungen darauf hinweist, dass er nicht für das Wohl der ganzen Welt verantwortlich ist, dann spielt er auf genau diese legitime Indifferenz an. Effekt dieser ,funktionalen Entlastung' (vgl. Luhmann 1975a: 145 ff.) ist jetzt nicht eine Unabhängigkeit der Wirtschaft als eigenes Funktionssystem, sondern das genaue Gegenteil. Da sich die Wirtschaft ,nur' noch auf das Prinzip der Profitmaximierung konzentrieren kann und zum Beispiel nicht mehr - wie zu Beginn des europäischen Kapitalismus und teilweise heute noch in Entwicklungsländern - mit Privatarmeen selbst für den Schutz ihres konstanten und variablen Kapitals sorgen muss, wird die Abhängigkeit vom Funktionieren der Polizei groß. Da sich kapitalistische Unternehmen nicht mehr ihre ,eigenen Richter' halten, sind sie auf eine Autonomie des Rechtswesens angewiesen. Wenn die Politik wie in Russland das Rechtswesen für ihre Zwecke vereinnahmt und Unternehmer mit fadenscheinigen Gründen verurteilen lassen kann, kann die Entwicklung einer eigenständigen kapitalisti-

\footnotetext{
22 Diese Lesart von Marx findet sich zwischen den Zeilen bei Parsons' und Smelsers Arbeit Economy and Society (vgl. Parsons, Smelser 1957: 36 ff.).

${ }^{23}$ André Kieserling danke ich dafür, dass er mich auf das entsprechende Argument bei Luhmann aufmerksam gemacht hat. Das Argument habe ich in Kühl (2004: 39 ff.) ausführlicher entwickelt. Eine frühe Kritik an diesem Konzept aus marxistischer Sicht findet sich bei Türk (1987: 52 ff.).
} 
schen Wirtschaft in Schwierigkeiten kommen. Es gibt aus dieser Perspektive keinen Grund, einem Funktionssystem eine besondere Prominenz zuzuweisen. Nicht nur die kapitalistische Wirtschaft ist von einem funktionierenden politischen System, einer lebhaften Wissenschaftslandschaft und einem ausgefeilten Rechtssystem abhängig, sondern auch die Politik muss sich auf ein funktionierendes Wirtschaftssystem und eine stabile Rechtsordnung verlassen. Ein Theoretiker funktionaler Differenzierung würde den marxistischen Staatstheoretikern entgegenhalten, dass sie das Problem von Abhängigkeiten gesellschaftlicher Teilbereiche wie Politik und Recht von der Wirtschaft richtig erkannt haben, dass es aber keinen Grund gibt, dieses Abhängigkeitsverhältnis auf die Wirtschaft zu begrenzen. ${ }^{24}$

Was bedeutet dieses Argument zugespitzt für das Verhältnis von Marxismus und Wirtschaftssoziologie? Aus meiner Sicht entfaltet der Marxismus seine Stärken, wenn es um die Behandlung wirtschaftssoziologischer Fragen im engeren Sinne geht. Für eine Programmatik des ,bringing society back in' müsste aber vermutlich auf andere Theorien zurückgegriffen werden.

\section{Literatur}

Abbott, Andrew, 2005: Sociology of Work and Occupations. S. 207-330 in: Smelser, Neil J. und Richard Swedberg (Hg.), The Handbook of Economic Sociology. 2. Aufl., Princeton: Princeton University Press.

Adorno, Theodor W., 1969: Einleitungsvortrag zum 16. Deutschen Soziologentag. S. 12-28 in: Adorno, Theodor W., Deutscher Soziologentag und Deutsche Gesellschaft für Soziologie (Hg.), Spätkapitalismus oder Industriegesellschaft? Verhandlungen des 16. Deutschen Soziologentages. Stuttgart: Enke.

Aglietta, Michel, 1979: A Theory of Capitalist Regulation. The US Experience. London: NLB.

Aglietta, Michel, 2000: Ein neues Akkumulationsregime. Die Regulationstheorie auf dem Prüfstand. Hamburg: VSA.

Aglietta, Michel, 2002: Interview: Mutationen des Kapitalismus. Eine schizophrene Arbeitsgesellschaft? Sozialismus 29: S. 13-19.

Aron, Raymond, 1964: Die industrielle Gesellschaft. 18 Vorlesungen. Frankfurt/M.: Fischer.

Baecker, Dirk, 2006: Wirtschaftssoziologie. Bielefeld: transcript.

Bahrdt, Hans Paul, 1982: Die Industriesoziologie - eine "spezielle Soziologie“? S. 11-15 in: Schmidt, Gert, Hans-Joachim Braczyk und Jost von dem Knesebeck (Hg.), Materialien zur Industriesoziologie. Sonderheft 24 der Kölner Zeitschrift für Soziologie und Sozialpsychologie. Opladen: Westdeutscher Verlag.

Baran, Paul A. und Paul M. Sweezy, 1966: Monopoly Capital. An Essay on the American Economic and Social Order. New York: Monthly Review Press.

\footnotetext{
${ }^{24}$ Die Theorie funktionaler Differenzierung gibt bisher nur erste Ansatzpunkte, wie trotzdem ein ,funktionales Primat' der Ökonomie zu denken wäre. In der durch Marx geprägten Soziologie wird das Primat der Ökonomie einerseits über eine besondere Rolle der Wirtschaft bei der Entwicklung der modernen Gesellschaft und andererseits über eine stärkere Machtposition der Wirtschaft gegenüber anderen gesellschaftlichen Teilbereichen bestimmt. In ihrer - zugegebenermaßen - karikaturellen Variante stellt man sich vor, wie das Kapital die Massenmedien kontrolliert, wie die Politiker durch die Wirtschaft geführt werden oder die Wissenschaft gezwungen wird, sich an ökonomischen Vorgaben zu orientieren. Luhmann dagegen versteht unter dem Primat eines Funktionssystems keine Herrschaft eines Funktionssystems über ein anderes. Primat heißt bei ihm vielmehr, dass die Entwicklung einer Gesellschaft „in erster Linie von einem ihrer funktional notwendigen Teilsysteme abhängt“ und die anderen Funktionssysteme ihre Probleme durch dieses Funktionssystem vordefiniert erhalten (Luhmann 1975b: 96; vgl. Luhmann 1997: 747).
} 
Barnard, Chester I., 1938: The Functions of the Executive. Cambridge: Harvard University Press.

Beck, Ulrich, 1986: Risikogesellschaft. Auf dem Weg in eine andere Moderne. Frankfurt/M.: Suhrkamp.

Beck, Ulrich, 1997: Was ist Globalisierung? Irrtümer des Globalismus - Antworten auf Globalisierung. 4. Aufl., Frankfurt/M.: Suhrkamp.

Beck, Ulrich, 2000: Wohin führt der Weg, der mit dem Ende der Vollbeschäftigungsgesellschaft beginnt?

S. 7-66 in: Beck, Ulrich (Hg.), Die Zukunft von Arbeit und Demokratie. Frankfurt/M.: Suhrkamp.

Beckenbach, Niels, 1991: Industriesoziologie. Berlin: de Gruyter.

Berger, Johannes, 1999: Was behauptet die Marxsche Klassentheorie und was ist davon zu halten? S. 77-

104 in: Berger, Johannes (Hg.), Die Wirtschaft der modernen Gesellschaft. Strukturprobleme und Zukunftsperspektiven. Frankfurt/M.: Campus.

Berle, Adolf A. und Gardiner C. Means, 1934: The Modern Corporation and Private Property. New York: Macmillan.

Boltanski, Luc und Ève Chiapello, 1999: Le nouvel esprit du capitalisme. Paris: Gallimard.

Booth, David, 1985: Marxism and Development Sociology. Interpreting the Impasse. World Development 13: S. 761-787.

Borrus, Michael und John Zysman, 1998: Globalization with Borders. The Rise of „Wintelism“ as the Future of Industrial Competition. S. 27-62 in: Zysman, John und Andrew Schwartz (Hg.), Enlarging Europe. The Industrial Foundations of a New Political Reality. Berkeley: University of California Press.

Boyer, Robert, 2005: How and Why Capitalisms Differ. Economy and Society 34: S. 509-557.

Braverman, Harry, 1974: Labor and Monopoly Capital. The Degradation of Work in the Twentieth Century. 3. Print, New York: Monthly Review Press.

Brenner, Robert, 1976: The Origins of Capitalist Development. A Critique of Neo-Smithian Marxism. New Left Review 104: S. 25-92.

Brewer, Anthony, 1980: Marxist Theories of Imperialism. A Critical Survey. London: Routledge \& Kegan Paul.

Burawoy, Michael, 1979: Manufacturing Consent. Changes in the Labor Process Under Monopoly Capitalism. Chicago: University of Chicago Press.

Buß, Eugen, 1985: Lehrbuch der Wirtschaftssoziologie. Berlin: Walter de Gruyter.

Chirot, Daniel und Thomas Hall, 1982: World-System Theory. Annual Review of Sociology 8: S. 81-106.

Commons, John R., 1924: Legal Foundation of Capitalism. New York: Macmillan.

Deppe, Frank, 1971: Das Bewußtsein der Arbeiter. Studien zur politischen Soziologie des Arbeiterbewußtseins. Köln: Pahl-Rugenstein.

ECLA, United Nations Economic Commission for Latin America, 1970: Development Problems in Latin America. Austin: University of Texas Press.

Engels, Friedrich, 1969: Der Ursprung der Familie, des Privateigentums und des Staates. S. 30-173 in: Marx, Karl und Friedrich Engels, MEW 21. Berlin: Dietz.

Esping-Andersen, Gøsta, 1990: The Three Worlds of Welfare Capitalism. Cambridge: Polity Press.

Fligstein, Neil, 2005: The Political and Economic Sociology of International Economic Arrangements. S. 183-204 in: Smelser, Neil J. und Richard Swedberg (Hg.), The Handbook of Economic Sociology. 2. Aufl., Princeton: Princeton University Press.

Ford, Henry, 1923: Mein Leben und Werk. 2. Aufl., Leipzig: List.

Frank, André Gunder, 1998: ReOrient. Global Economy in the Asian Age. Berkeley: University of California Press.

Frank, André Gunder, 2000: The Development of Underdevelopment. S. 159-168 in: Roberts, J. Timmons und Amy Hite (Hg.), From Modernization to Globalization. Perspectives on Development and Social Change. Malden: Blackwell.

Friedman, Andrew L., 1977: Industry and Labour. Class Struggle at Work and Monopoly Capitalism. London: Macmillan. 
Friedmann, Georges, 1959: Grenzen der Arbeitsteilung. Frankfurt/M.: EVA.

Fürstenberg, Friedrich, 1970: Wirtschaftssoziologie. 2., neubearb. u. erg. Aufl., Berlin: de Gruyter.

Geiger, Theodor, 1949: Die Klassengesellschaft im Schmelztiegel. Köln: Kiepenheuer.

Gereffi, Gary, 2005: The Global Economy: Organization, Governance, and Development. S. 160-182 in: Smelser, Neil J. und Richard Swedberg (Hg.), The Handbook of Economic Sociology. 2. Aufl., Princeton: Princeton University Press.

Glick, Mark und Robert Brenner, 1999: Der Regulationsansatz: Theorie und Geschichte. S. 37-130 in: Marxismus, Arbeitsgruppe (Hg.), Kapitalistische Entwicklung und Krisen. Eine marxistische Kritik der „Regulationstheorie“. Wien: AGM.

Gramsci, Antonio, 1999: Heft 22. Amerikanismus und Fordismus S. 2061-2101 in: Gramsci, Antonio, Gefängnishefte. Kritische Gesamtausgabe, Bd. 9, Hefte 22 bis 29. Hamburg: Argument.

Haber, Samuel, 1964: Efficiency and Uplift. Scientific Management in the Progressive Era 1890-1920. Chicago: University of Chicago Press.

Hack, Lothar, 2005: Auf der Suche nach der verlorenen Totalität. Von Marx' kapitalistischer Gesellschaftstransformation zu Wallersteins Analyse der "Weltsysteme“? S. 120-158 in: Heintz, Bettina, Richard Münch und Hartmann Tyrell (Hg.), Weltgesellschaft. Theoretische Zugänge und empirische Problemlagen. Sonderheft der Zeitschrift für Soziologie Stuttgart: Lucius \& Lucius.

Hall, Peter A. und David Soskice, 2001: An Introduction to Varieties of Capitalism. S. 1-70 in: Hall, Peter A. und David Soskice (Hg.), Varieties of Capitalism. The Institutional Foundations of Comparative Advantage. Oxford: Oxford University Press.

Hall, Peter A. und David Soskice, 2003: Varieties of Capitalism and Institutional Change: A Response to Three Critics. Comparative European Politics 1: S. 241-250.

Hall, Peter A. und Daniel W. Gingerich, 2004: Varieties of Capitalism and the Institutional Complementaries in Macroeconomy: An Empirical Analysis. Discussion Paper 04/5. Köln: Max-Planck-Institut für Gesellschaftsforschung.

Hardt, Michael und Antonio Negri, 2003: Empire. Die neue Weltordnung. Frankfurt/M.: Campus.

Heigl, Miriam, 2005: Auf dem Weg zur finalen Krise des Kapitalismus? Weltsystemtheoretische Beiträge zur neuen Debatte des Imperialismus. Prokla 139: S. 267-286.

Heinemann, Klaus, 1987: Probleme der Konstituierung einer Wirtschaftssoziologie. S. 7-39 in: Heinemann, Klaus (Hg.), Soziologie wirtschaftlichen Handelns. Sonderheft 28 der Kölner Zeitschrift für Soziologie und Sozialpsychologie. Opladen: Westdeutscher Verlag.

Heintz, Peter, 1982: Die Weltgesellschaft im Spiegel von Ereignissen. Diessenhofen: Rüegger.

Helmedag, Fritz, 1992: Warenproduktion mittels Arbeit. Zur Rehabilitation des Wertgesetzes. Marburg: Metropolis.

Hillmann, Karl-Heinz, 1988: Allgemeine Wirtschaftssoziologie. Eine grundlegende Einführung. München: Vahlen.

Hirsch, Joachim und Roland Roth, 1986: Das neue Gesicht des Kapitalismus. Vom Fordismus zum PostFordismus. Hamburg: VSA.

Hirsch, Joachim, 1995: Der nationale Wettbewerbsstaat. Staat, Demokratie und Politik im globalen Kapitalismus. Berlin: Edition.

Hirsch, Joachim, 2005: Materialistische Staatstheorie. Transformationsprozesse des kapitalistischen Staatensystems. Hamburg: VSA.

Hirsch-Kreinsen, Hartmut, 2005: Wirtschafts- und Industriesoziologie. Grundlagen, Fragestellungen, Themenbereiche. Weinheim: Juventa.

Hofmann, Werner, 1971: Arbeitswertlehre und kapitalistische Entwicklung. München: Linskdruck.

Hopkins, Terence K. und Immanuel Wallerstein, 1982: Patterns of Development of the Modern WorldSystem. S. 41-82 in: Hopkins, Terence K. und Immanuel Wallerstein (Hg.), World-Systems Analysis. Theory and Methodology. London: Sage. 
Hübner, Kurt und Birgit Mahnkopf, 1988: Einleitung. S. 7-28 in: Mahnkopf, Birgit (Hg.), Der gewendete Kapitalismus. Kritische Beiträge zur Theorie der Regulation. Münster: Westfälisches Dampfboot.

Jansen, Dorothea, 2005: Von Organisationen und Märkten zur Wirtschaftssoziologie. S. 227-258 in: Faust, Michael, Maria Funder und Manfred Moldaschl (Hg.), Die "Organisation“ der Arbeit. München: Hampp.

Jessop, Bob, 2003: Postfordismus und wissensbasierte Ökonomie. Eine Reinterpretation des Regulationsansatzes. S. 89-113 in: Brand, Ulrich und Werner Raza (Hg.), Fit für den Postfordismus? Theoretischpolitische Perspektiven des Regulationsansatzes. Münster: Westfälisches Dampfboot.

Jürgens, Ulrich, 1984: Die Entwicklung von Macht, Herrschaft und Kontrolle im Betrieb als politischer Prozess. S. 11-57 in: Jürgens, Ulrich und Frieder Naschold (Hg.), Arbeitspolitik. Materialien zum Zusammenhang von politischer Macht, Kontrolle und betrieblicher Organisation der Arbeit. Sonderheft 5 des Leviathan. Opladen: Westdeutscher Verlag.

Kern, Horst und Michael Schumann, 1984: Das Ende der Arbeitsteilung? Rationalisierung in der industriellen Produktion. München: Beck.

Kern, Horst, 1997: Industriesoziologie weit gedacht. S. 29-44 in: Bieber, Daniel (Hg.), Technikentwicklung und Industriearbeit. Industrielle Produktionstechnik zwischen Eigendynamik und Nutzerinteressen. Frankfurt/M.: Campus.

Kieserling, André, 2006: Klasse und Klassengesellschaft: Zur Entkopplung zweier Begriffe S. 435-436 in: Rehberg, Karl-Siegbert (Hg.), Soziale Ungleichheit, kulturelle Unterschiede. Verhandlungen des 32. Kongresses der Deutschen Gesellschaft für Soziologie in München 2004. Frankfurt/M.: Campus.

King, Lawrence und Iván Szelényi, 2005: Post-Communist Economic Systems. S. 205-229 in: Smelser, Neil J. und Richard Swedberg (Hg.), The Handbook of Economic Sociology. 2. Aufl., Princeton: Princeton University Press.

Koch, Max, 1994: Vom Strukturwandel einer Klassengesellschaft. Theoretische Diskussion und empirische Analyse. Münster: Westfälisches Dampfboot.

Kocka, Jürgen, 1983: Lohnarbeit und Klassenbildung. Arbeiter und Arbeiterbewegung in Deutschland 1800-1875. Berlin: Dietz Nachfolge.

Kühl, Stefan, 2003: Exit. Wie Risikokapital die Regeln der Wirtschaft verändert. Frankfurt/M.: Campus.

Kühl, Stefan, 2004: Arbeits- und Industriesoziologie. Bielefeld: transcript.

Kurz, Robert, 2001: Schwarzbuch Kapitalismus. Ein Abgesang auf die Marktwirtschaft. München: Ullstein.

Laclau, Ernesto, 1971: Feudalism and Capitalism in Latin America. New Left Review 67: S. 166-190.

Landshut, Siegfried, 1956: Die Gegenwart im Lichte der Marxschen Lehre. Hamburger Jahrbuch für Wirtschafts- und Gesellschaftspolitik 1: S. 42-55.

Lipietz, Alain, 1985: Akkumulation, Krisen und Auswege aus der Krise. Einige methodologische Anmerkungen zum Begriff der „Regulation“. Prokla 58: S. 109-137.

Lipietz, Alain und Hans-Peter Krebs, 1998: Nach dem Ende des „Goldenen Zeitalters“. Regulation und Transformation kapitalistischer Gesellschaften. Berlin: Argument.

Littler, Craig R., 1990: The Labour Process Debate. A Theoretical Review 1974-1988. in: Knights, David und Hugh Willmott (Hg.), Labour Process Theory. Basingstoke: Macmillan.

Luhmann, Niklas, 1965: Grundrechte als Institution. Ein Beitrag zur politischen Soziologie. Berlin: Duncker \& Humblot.

Luhmann, Niklas, 1971: Die Weltgesellschaft. Archiv für Rechts- und Sozialphilosophie 57: S. 1-35.

Luhmann, Niklas, 1975a: Legitimation durch Verfahren. 2. Aufl., Darmstadt: Luchterhand.

Luhmann, Niklas, 1975b: Selbst-Thematisierung des Gesellschaftssystems. S. 70-102 in: Luhmann, Niklas, Soziologische Aufklärung, Bd. 2. Aufsätze zur Theorie sozialer Systeme. Opladen: Westdeutscher Verlag.

Luhmann, Niklas, 1981: Organisation im Wirtschaftssystem. S. 390-141 in: Luhmann, Niklas, Soziologische Aufklärung, Bd. 3. Soziales System, Gesellschaft, Organisation. Opladen: Westdeutscher Verlag. 
Luhmann, Niklas, 1985: Zum Begriff der sozialen Klasse. S. 119-163 in: Luhmann, Niklas, Soziale Differenzierung. Zur Geschichte einer Idee. Opladen: Westdeutscher Verlag.

Luhmann, Niklas, 1997: Die Gesellschaft der Gesellschaft. Frankfurt/M.: Suhrkamp.

Lutz, Burkart und Gert Schmidt, 1977: Industriesoziologie. in: König, René (Hg.), Handbuch der empirischen Sozialforschung. 2., völlig neubearb. Aufl., München: dtv.

Lutz, Burkart, 1989: Der kurze Traum immerwährender Prosperität. Eine Neuinterpretation der industriell-kapitalistischen Entwicklung im Europa des 20. Jahrhunderts. Frankfurt/M.: Campus.

Makler, Harry, Arnaud Sales und Neil J. Smelser, 1982: Recent Trends in Theory and Methodology in the Study of Economy and Society. S. 147-171 in: Bottomore, Tom, Stefan Nowak und Magdalena Sokolowska (Hg.), Sociology. The State of the Art. London: Sage.

Mallet, Serge, 1969: La nouvelle classe ouvrière. Paris: Seuil.

Marx, Karl, 1959: Das Elend der Philosophie. Orig. v. 1847. S. 63-182 in: Marx, Karl und Friedrich Engels, MEW 4. 2. Aufl., Berlin: Dietz.

Marx, Karl und Friedrich Engels, 1959: Das Manifest der kommunistischen Partei. Orig. v. 1848. S. 459-493 in: Marx, Karl und Friedrich Engels, MEW 4. 2. Aufl., Berlin: Dietz.

Marx, Karl, 1960: Der achtzehnte Brumaire des Louis Bonaparte. Orig. v. 1851. S. 111-207 in: Marx, Karl und Friedrich Engels, MEW 8. Berlin: Dietz.

Marx, Karl, 1961: Einleitung (zur Kritik der Politischen Ökonomie). Erstausg. v. 1939 (aus Nachlass). S. 615-641 in: Marx, Karl und Friedrich Engels, MEW 13. Berlin: Dietz.

Marx, Karl, 1961: Zur Kritik der Politischen Ökonomie. Orig. v. 1859. S. 3-160 in: Marx, Karl und Friedrich Engels, MEW 13. Berlin: Dietz.

Marx, Karl, 1962: Das Kapital. Kritik der politischen Ökonomie, Bd. 1. (MEW 23). Orig. v. 1867, Berlin: Dietz.

Marx, Karl, 1964: Das Kapital. Kritik der politischen Ökonomie, Bd. 3 (MEW 25). Orig. v. 1894, Berlin: Dietz.

Meyer, John W., 1980: The World Polity and the Authority of the Nation-State. S. 109-137 in: Bergesen, Albert (Hg.), Studies of the Modern World-System. New York: Academic Press.

Moldaschl, Manfred und Wolfgang G. Weber, 1998: The „Three Waves“ of Industrial Group Work. Historical Reflections on Current Research on Group Work. Human Relations 51: S. 347-388.

Mooser, Josef, 1983: Auflösung proletarischer Milieus. Klassenbindung und Individualisierung in der Arbeiterschaft vom Kaiserreich bis in die Bundesrepublik Deutschland. Soziale Welt 34: S. 270305.

Münch, Richard, 2004: Soziologische Theorie, Bd. 3. Gesellschaftstheorie. Frankfurt/M.: Campus.

Offe, Claus, 1972: Strukturprobleme des kapitalistischen Staates. Aufsätze zur politischen Soziologie. Frankfurt/M.: Suhrkamp.

Offe, Claus, 1984: Arbeit als soziologische Schlüsselkategorie? S. 13-43 in: Offe, Claus (Hg.), „Arbeitsgesellschaft": Strukturprobleme und Zukunftsperspektiven. Frankfurt/M.: Campus.

Offe, Claus, 2006: Strukturprobleme des kapitalistischen Staates. Aufsätze zur Politischen Soziologie. Veränd. Neuausg., Frankfurt/M.: Campus.

Ortmann, Günther, 1994: Dark Stars - Institutionelles Vergessen in der Industriesoziologie. S. 85-118 in: Beckenbach, Niels und Werner van Treeck (Hg.), Umbrüche gesellschaftlicher Arbeit. Soziale Welt, Sonderband 9. Göttingen: Schwartz.

Parsons, Talcott und Neil J. Smelser, 1957: Economy and Society. A Study in the Integration of Economic and Social Theory. 2. Aufl., London: Routledge \& Kegan Paul.

Polanyi, Karl, 1995: The Great Transformation. Politische und ökonomische Ursprünge von Gesellschaften und Wirtschaftssystemen. 3. Aufl., Engl. Orig. v. 1944, Frankfurt/M.: Suhrkamp.

Prebisch, Raúl, 1959: Commercial Policy in the Underdeveloped Countries. American Economic Review 49: S. 251-273. 
Prebisch, Raúl, 1962: The Economic Development of Latin America and its Principal Problems. Economic Bulletin for Latin America 7: S. 1-22.

Roth, Karl Heinz, 1994: Die Wiederkehr der Proletarität und die Angst der Linken. S. 11-36 in: Roth, Karl Heinz (Hg.), Die Wiederkehr der Proletarität. Dokumentation der Debatte. Köln: ISP.

Röttger, Bernd, 2003: Verlassene Gräber und neue Pilger an der Grabstätte. Eine neo-regulationistische Perspektive. S. 18-42 in: Brand, Ulrich und Werner Raza (Hg.), Fit für den Postfordismus? Theoretisch-politische Perspektiven des Regulationsansatzes. Münster: Westfälisches Dampfboot.

Scharpf, Fritz W., 1989: Politische Steuerung und politische Institutionen. Politische Vierteljahresschrift 30: S. 10-21.

Scharpf, Fritz W., 1992: Koordination durch Verhandlungssysteme. S. 51-96 in: Benz, Arthur, Fritz W. Scharpf und Reinhard Zintl (Hg.), Horizontale Politikverflechtung. Zur Theorie von Verhandlungssystemen. Frankfurt/M.: Campus.

Schelsky, Helmut, 1965: Auf der Suche nach Wirklichkeit. Gesammelte Aufsätze. Düsseldorf: Diederichs. Simon, Herbert A., 1957: Models of Man. Social and Rational. New York: Wiley \& Sons.

Smelser, Neil J. und Richard Swedberg, 2005: Introducing Economic Sociology. S. 3-26 in: Smelser, Neil J. und Richard Swedberg (Hg.), The Handbook of Economic Sociology. 2. Aufl., Princeton: Princeton University Press.

Springer, Roland, 1999: Rückkehr zum Taylorismus? Arbeitspolitik in der Automobilindustrie am Scheideweg. Frankfurt/M.: Campus.

Stearns, Linda B. und Mark S. Mizruchi, 2005: Banking and Financial Markets. S. 284-306 in: Smelser, Neil J. und Richard Swedberg (Hg.), The Handbook of Economic Sociology. 2. Aufl., Princeton: Princeton University Press.

Swedberg, Richard, 1987: Economic Sociology: Past and Present. Current Sociology 35: S. 1-221.

Tanner, Jakob, 1999: Fordismus. S. 580-587 in: Haug, Wolfgang F. (Hg.), Historisch-kritisches Wörterbuch des Marxismus, Bd. 4. Hamburg: Argument.

Türk, Klaus, 1987: Einführung in die Soziologie der Wirtschaft. Stuttgart: Teubner.

Tyrell, Hartmann, 1978: Anfragen an die Theorie gesellschaftlicher Differenzierung. Zeitschrift für Soziologie 7: S. 175-193.

Vahrenkamp, Richard, 1976: Taylors Lehren - ein Mittelklassentraum. Überlegungen zu einem Rätsel. S. 14-26 in: Michel, Karl Markus (Hg.), Arbeitsorganisation. Ende des Taylorismus. Kursbuch 43. Berlin: Rotbuch.

Wallerstein, Immanuel, 1974a: Dependence in an Interdependent World. The Limited Possibilities of Transformation within the Capitalist World Order. African Studies Review 17: S. 1-26.

Wallerstein, Immanuel, 1974b: The Modern World-System. Capitalist Agriculture and the Origins of the European World-Economy in the Sixteenth Century. New York: Academic Press.

Wallerstein, Immanuel, 1976: Modernization: Requescat in Pace. S. 131-135 in: Coser, Lewis A. und Otto N. Larsen (Hg.), The Uses of Controversy in Sociology. New York: The Free Press.

Wallerstein, Immanuel, 1984a: Der historische Kapitalismus. Berlin: Argument.

Wallerstein, Immanuel, 1984b: The Politics of the World-Economy. The States, the Movements, and the Civilizations. Cambridge: Cambridge University Press.

Wallerstein, Immanuel, 1991: Development: Lodestar or Illusion? S. 104-124 in: Wallerstein, Immanuel, Unthinking Social Science. The Limits of Nineteenth-Century Paradigms. Cambridge: Polity Press.

Wallerstein, Immanuel, 2003: The Decline of American Power. The U.S. in a Chaotic World. New York: New Press.

Wark, McKenzie, 1991: From Fordism to Sonyism. New Formations 15: S. 43-54.

Weber, Hajo und Martina Wegge, 2003: Zur Soziologie der Wirtschaft der Gesellschaft - Theorie, Forschung und Perspektiven der Wirtschaftssoziologie. S. 547-562 in: Orth, Barbara, Thomas Schwietring und Johannes Weiß (Hg.), Soziologische Forschung. Stand und Perspektiven. Opladen: Leske + Budrich. 
Weber, Max, 1972: Wirtschaft und Gesellschaft. Grundriß der verstehenden Soziologie. 5., rev. Aufl., Orig. v. 1922, Tübingen: Mohr Siebeck.

Weiss, Hilde, 1936: Die „Enquete Ouvrière“ von Karl Marx. Zeitschrift für Sozialforschung 5: S. 76-98.

Wright, Erik O., 1978: Class, Crisis and the State. London: New Left Books.

Wright, Erik O., 1985: Classes. London: Verso.

Wuthnow, Robert, 2005: New Directions in the Study of Religion and Economic Life. S. 603-626 in: Smelser, Neil J. und Richard Swedberg (Hg.), The Handbook of Economic Sociology. 2. Aufl., Princeton: Princeton University Press. 